\title{
Analysis of a Decoupled Time-Stepping Scheme for Evolutionary Micropolar Fluid Flows
}

\author{
S. S. Ravindran \\ Department of Mathematical Sciences, 201M Shelby Center for Science and Technology, \\ The University of Alabama in Huntsville, Huntsville, AL 35899, USA
}

Correspondence should be addressed to S. S. Ravindran; ravinds@uah.edu

Received 28 April 2016; Accepted 26 June 2016

Academic Editor: William J. Layton

Copyright (c) 2016 S. S. Ravindran. This is an open access article distributed under the Creative Commons Attribution License, which permits unrestricted use, distribution, and reproduction in any medium, provided the original work is properly cited.

Micropolar fluid model consists of Navier-Stokes equations and microrotational velocity equations describing the dynamics of flows in which microstructure of fluid is important. In this paper, we propose and analyze a decoupled time-stepping algorithm for the evolutionary micropolar flow. The proposed method requires solving only one uncoupled Navier-Stokes and one microrotation subphysics problem per time step. We derive optimal order error estimates in suitable norms without assuming any stability condition or time step size restriction.

\section{Introduction}

Micropolar fluid model theory considers the interaction between the fluid motion and rotational motion of microparticles suspended in a viscous medium when the deformation of microparticles is ignored. Numerous experimental and numerical studies have indicated that the micropolar fluid theory better describes micro- and nanoflows than the classical Naiver-Stokes theory. Understanding microscale fluid flow phenomena is important in order to effectively design and fabricate microchannels and chambers for microfluidic systems [1]. Growing interest in microscale flow phenomena is also due to the miniaturization of fluid devices for controlling flows in micromachines. Numerical predictions reported in [2] and experimental studies reported in [3-7] show that micropolar fluid models better represent the behavior of flows in microfluidic systems compared to the Navier-Stokes equations. In the experimental work reported in [7], fluids containing minute polymeric additives indicate considerable reduction of the skin friction which can be related to the presence of antisymmetric and coupled stresses in micropolar fluids leading to an increase in the energy dissipation.

There are numerous papers devoted to the mathematical analysis of micropolar fluid flows such as the existence and uniqueness of solutions to micropolar flow equations; see [813]. In [14-16], optimal control problems associated with micropolar fluids are studied from the theoretical point of view. Stability problems for micropolar fluids are investigated in $[17,18]$. It has also been the subject of many computational simulation based investigations [2,19-22]. These works mainly focus on the numerical solution of micropolar fluid equations modeling various applied problems such as HagenPoiseuille flow and nano/microfluid system [23, 24]. Micropolar fluid models for real and nontrivial flow problems would involve a system of nineteen partial differential equations in nineteen unknowns, therefore computationally very challenging. Despite these challenges in computing micropolar fluid flow, there are very few studies in the literature on numerical analysis and algorithms for efficient computation of micropolar fluid flows. In [25], a numerical scheme based on projection method in time and finite-difference in space is incorporated to solve unsteady incompressible micropolar fluid flow problems. In [26], convergence rate of Galerkin spectral spatial approximation for the micropolar fluid model is studied.

In the present work, we propose and study a decoupled time-stepping scheme for the evolutionary micropolar fluid flow model. It uses a semi-implicit Crank-Nicolson scheme 
that combines an implicit treatment of the second derivative terms, a semi-implicit second-order extrapolation of the nonlinear convective terms, and explicit treatment of the coupling terms. The proposed scheme solves the NavierStokes equations and the microrotational velocity equations separately in each time step without iteration. We derive optimal order error estimates of the scheme without any stability condition or time step size restriction.

An outline of the paper is as follows. In Section 2, we present the governing equations and some preliminary materials. In Section 3, we propose a decoupled Crank-Nicolson time-stepping scheme using extrapolation in time and prove that the proposed decoupled scheme yields the second-order convergence in the temporal direction. Numerical tests are reported in Section 4.

\section{Micropolar Fluid System}

2.1. Formulation of the Problem. Incompressible flow of micropolar fluids is modeled by the system; see, for example, [27-29]. Given $\mathbf{f}_{1}, \mathbf{f}_{2}, \mathbf{g}$, and $\mathbf{q}$ and time $T>0$, find $\mathbf{u}$ : $\Omega \times[0, T] \rightarrow \mathbb{R}^{d}, p: \Omega \times[0, T] \rightarrow \mathbb{R}$, and $\mathbf{w}: \Omega \times[0, T] \rightarrow \mathbb{R}^{d}$ such that

$$
\begin{aligned}
& \partial_{t} \mathbf{u}-\left(\nu+v_{r}\right) \Delta \mathbf{u}+(\mathbf{u} \cdot \nabla) \mathbf{u}+\nabla p=2 \nu_{r} \nabla \times \mathbf{w}+\mathbf{f}_{1} \\
& \text { in } \Omega \times(0, T] \text {, } \\
& \partial_{t} \mathbf{w}-\left(c_{a}+c_{d}\right) \Delta \mathbf{w}-\left(c_{0}+c_{d}-c_{a}\right) \nabla(\nabla \cdot \mathbf{w})+(\mathbf{u} \cdot \nabla) \mathbf{w} \\
& +4 v_{r} \mathbf{w}=2 v_{r} \nabla \times \mathbf{u}+\mathbf{f}_{2} \quad \text { in } \Omega \times(0, T], \\
& \nabla \cdot \mathbf{u}=0 \quad \text { in } \Omega \times(0, T] \text {, }
\end{aligned}
$$

where $\mathbf{u}$ is the fluid velocity, $\mathbf{w}$ the microrotation field interpreted as the angular velocity field of rotation of particles, and $p$ the fluid kinematic pressure. Notice that the microrotation vector $\mathbf{w}$ is equal neither to the flow vorticity $\nabla \times \mathbf{u}$ nor to average flow angular velocity $(1 / 2) \nabla \times \mathbf{u}$. The fields $\mathbf{f}_{1}$ and $\mathbf{f}_{2}$ are the external body force and moment (torque), respectively. The positive constants $\nu, v_{r}, c_{0}, c_{a}$, and $c_{d}$ represent viscosity coefficients, $v$ is the usual Newtonian viscosity, and $\nu_{r}$ is the microrotation viscosity. Moreover, the constants $c_{0}, c_{d}$, and $c_{a}$ satisfy the inequality $c_{0}+c_{d}>$ $c_{a}$. The system is supplemented by the Dirichlet boundary conditions,

$$
\begin{array}{r}
\mathbf{u}=\mathbf{g}, \\
\mathbf{w}=\mathbf{q}
\end{array}
$$

$$
\text { on } \Gamma \times(0, T] \text {, }
$$

and the initial conditions,

$$
\begin{aligned}
\mathbf{u}(\mathbf{x}, 0) & =\mathbf{u}_{0}(\mathbf{x}), \\
\mathbf{w}(\mathbf{x}, 0) & =\mathbf{w}_{0}(\mathbf{x})
\end{aligned}
$$

in $\Omega$.

Here $\Omega$ is a bounded, Lipschitz domain in $\mathbb{R}^{d}(d=3)$ and $\int_{\Gamma} \mathbf{g} \cdot \mathbf{n} d s=0$. Notice that $\mathbf{w}$ is a vector variable and the equations satisfied by its components $w_{i}$ are coupled via the second-order terms $\nabla(\nabla \cdot \mathbf{w})$ which may pose difficulty.

2.2. Weak Formulation of the Evolutionary Micropolar Fluid Model. For a Banach space $X$, we denote by $L^{p}(0, T ; X)$ the time-space function space endowed with the norm $\|w\|_{L^{p}(0, T ; X)}:=\left(\int_{0}^{T}\|w\|_{X}^{p} d t\right)^{1 / p}$ if $1 \leq p<\infty$ and

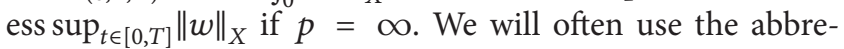
viated notation $L^{p}(X):=L^{p}(0, T ; X)$ for convenience. The symbol $C([0, T] ; X)$ denotes the set of continuous functions $u:[0, T] \rightarrow X$ endowed with the norm $\|u\|_{C(0, T ; X)}:=\max _{0 \leq t \leq T}\|u(t)\|_{X}$. For any integer $k \geq 1$, let $W^{k, p}(\Omega)$ be the Sobolev space of functions in $L^{p}(\Omega)$ with derivatives up to the $k$ th order endowed with the norm $\|\phi\|_{m, p}:=\left[\sum_{|\alpha| \leq m} \int_{\Omega}\left|\partial_{x}^{\alpha} \phi(\mathbf{x})\right|^{p} d x\right]^{1 / p}$, where $\partial_{x}^{\alpha} \phi(\mathbf{x}):=$ $\left(\partial^{|\alpha|} /\left(\partial_{x_{1}}^{\alpha_{1}} \cdots \partial_{x_{d}}^{\alpha_{d}}\right)\right) \phi(\mathbf{x}), \alpha:=\left(\alpha_{1}, \ldots, \alpha_{d}\right), \alpha_{i} \geq 0,|\alpha|:=$ $\sum_{i=1}^{d} \alpha_{i}$. We denote by $H^{k}(\Omega)$ the space $W^{k, 2}(\Omega)$, when $p=2$, and drop the subscripts $p(=2)$ in referring to the norm in $H^{k}(\Omega)$. Moreover, we will use the following simplified norm notations:

$$
\begin{aligned}
\|u\| & :=\|u\|_{L^{2}(\Omega)}, \\
\|u\|_{\infty} & :=\|u\|_{L^{\infty}(\Omega)} .
\end{aligned}
$$

We introduce the time discrete space $l^{p}(Z)$ associated with $L^{p}(0, T ; Z) ; l^{P}(Z)$ is the space of $Z$-valued sequences $w$ := $\left\{w_{n} ; n=1, \ldots, N\right\}$ with norm $\|\cdot\|_{l^{p}(Z)}$ defined by

$$
\|w\|_{l^{p}(Z)}:= \begin{cases}\left(\Delta t \sum_{n=1}^{N}\left\|w_{n}\right\|_{Z}^{p}\right)^{1 / p} & \text { if } 1 \leq p<\infty \\ \max _{1 \leq n \leq N}\left\|w_{n}\right\|_{Z} & \text { if } p=\infty .\end{cases}
$$

We define the spaces

$$
\begin{aligned}
\mathbf{H}_{g}^{1}(\Omega) & :=\left\{\mathbf{v} \in \mathbf{H}^{1}(\Omega):\left.\mathbf{v}\right|_{\Gamma}=\mathbf{g}\right\}, \\
\mathbf{V}_{g} & :=\left\{\mathbf{v} \in \mathbf{H}^{1}(\Omega):\left.\mathbf{v}\right|_{\Gamma}=\mathbf{g}, \nabla \cdot \mathbf{v}=0 \text { in } \Omega\right\},
\end{aligned}
$$

for $\mathbf{g} \in \mathbf{H}^{1 / 2}(\Gamma)$ satisfying $\int_{\Gamma} \mathbf{g} \cdot \mathbf{n} d s=0$,

$$
\mathbf{H}_{q}^{1}(\Omega):=\left\{\mathbf{v} \in \mathbf{H}^{1}(\Omega):\left.\mathbf{v}\right|_{\Gamma}=\mathbf{q}\right\}
$$

for $\mathbf{q} \in \mathbf{H}^{1 / 2}(\Gamma)$ and

$$
L_{0}^{2}(\Omega):=\left\{p \in L^{2}(\Omega): \int_{\Omega} p d \Omega=0\right\} .
$$

We often use the Sobolev inequality for $1 \leq q \leq 6$

$$
\|\mathbf{u}\|_{L^{q}(\Omega)} \leq c\|\mathbf{u}\|_{1} \quad \forall \mathbf{u} \in \mathbf{H}^{1}(\Omega)
$$

and the Poincaré inequality

$$
\|\mathbf{u}\| \leq \lambda\|\nabla \mathbf{u}\| \quad \forall \mathbf{u} \in \mathbf{H}_{0}^{1}(\Omega) .
$$

We recall also the Gagliardo-Nirenberg interpolation inequality

$$
\|\mathbf{u}\|_{1,3} \leq c\|\mathbf{u}\|_{1}^{1 / 2}\|\mathbf{u}\|_{2}^{1 / 2} \quad \forall \mathbf{u} \in \mathbf{H}^{2}(\Omega)
$$


and Agmon's inequality

$$
\|\mathbf{u}\|_{\infty} \leq c\|\mathbf{u}\|_{1}^{1 / 2}\|\mathbf{u}\|_{2}^{1 / 2} \quad \mathbf{u} \in \mathbf{H}^{2}(\Omega) \cap \mathbf{H}_{0}^{1}(\Omega),
$$

and see, for example, [30].

If we define the bilinear forms $a_{1}: \mathbf{H}^{1}(\Omega) \times \mathbf{H}^{1}(\Omega) \rightarrow \mathbb{R}$, $a_{2}: \mathbf{H}^{1}(\Omega) \times \mathbf{H}^{1}(\Omega) \rightarrow \mathbb{R}, d: \mathbf{H}^{1}(\Omega) \times \mathbf{H}^{1}(\Omega) \rightarrow \mathbb{R}$, and $b: \mathbf{H}^{1}(\Omega) \times L_{0}^{2}(\Omega) \rightarrow \mathbb{R}$ in the following way, for $\mathbf{u}_{1}, \mathbf{u}_{2}, \mathbf{u}_{3} \in$ $\mathbf{H}^{1}(\Omega)$ and $p \in L_{0}^{2}(\Omega)$ :

$$
\begin{aligned}
a_{1}\left(\mathbf{u}_{1}, \mathbf{u}_{2}\right):= & \left(v+v_{r}\right) \int_{\Omega} \nabla \mathbf{u}_{1} \cdot \nabla \mathbf{u}_{2} d \Omega, \\
a_{2}\left(\mathbf{u}_{1}, \mathbf{u}_{2}\right):= & \left(c_{a}+c_{d}\right) \int_{\Omega} \nabla \mathbf{u}_{1} \cdot \nabla \mathbf{u}_{2} d \Omega \\
& +\left(c_{0}+c_{d}-c_{a}\right) \int_{\Omega} \nabla \cdot \mathbf{u}_{1} \nabla \cdot \mathbf{u}_{2} d \Omega, \\
b\left(\mathbf{u}_{1}, p\right):= & -\int_{\Omega} p \nabla \cdot \mathbf{u}_{1} d \Omega, \\
d\left(\mathbf{w}_{1}, \mathbf{w}_{2}\right):= & 2 v_{r} \int_{\Omega} \nabla \times \mathbf{w}_{1} \cdot \mathbf{w}_{2} d \Omega,
\end{aligned}
$$

and define the trilinear form $c: \mathbf{H}^{1}(\Omega) \times \mathbf{H}^{1}(\Omega) \times \mathbf{H}^{1}(\Omega) \rightarrow \mathbb{R}$ as

$$
\begin{aligned}
& c\left(\mathbf{u}_{1}, \mathbf{u}_{2}, \mathbf{u}_{3}\right) \\
& \quad:=\frac{1}{2} \int_{\Omega}\left[\left(\mathbf{u}_{1} \cdot \nabla\right) \mathbf{u}_{2} \cdot \mathbf{u}_{3}-\left(\mathbf{u}_{1} \cdot \nabla\right) \mathbf{u}_{3} \cdot \mathbf{u}_{2}\right] d \Omega, \\
& \quad=\int_{\Omega}\left[\left(\mathbf{u}_{1} \cdot \nabla\right) \mathbf{u}_{2} \cdot \mathbf{u}_{3}+\frac{1}{2}\left(\nabla \cdot \mathbf{u}_{1}\right) \mathbf{u}_{2} \cdot \mathbf{u}_{3}\right] d \Omega,
\end{aligned}
$$

for all $\mathbf{u}_{1}, \mathbf{u}_{2}, \mathbf{u}_{3} \in \mathbf{H}^{1}(\Omega)$ with $\left(\mathbf{u}_{1} \cdot \mathbf{n}\right) \mathbf{u}_{2} \cdot \mathbf{u}_{3}=0$ on $\Gamma$, then the weak formulation of the micropolar fluid model is as follows: seek $(\mathbf{u}, \mathbf{w}) \in \mathbf{H}_{g}^{1}(\Omega) \times \mathbf{H}_{q}^{1}(\Omega)$ and $p \in L_{0}^{2}(\Omega)$ such that

$$
\begin{aligned}
& \left\langle\partial_{t} \mathbf{u}, \mathbf{v}\right\rangle+a_{1}(\mathbf{u}, \mathbf{v})+c(\mathbf{u}, \mathbf{u}, \mathbf{v})+b(\mathbf{v}, p) \\
& =d(\mathbf{w}, \mathbf{v})+\left\langle\mathbf{f}_{1}, \mathbf{v}\right\rangle \quad \forall \mathbf{v} \in \mathbf{H}_{0}^{1}(\Omega), \\
& b(\mathbf{u}, r)=0 \quad \forall r \in L_{0}^{2}(\Omega), \\
& \left\langle\partial_{t} \mathbf{w}, \boldsymbol{\phi}\right\rangle+a_{2}(\mathbf{w}, \boldsymbol{\phi})+c(\mathbf{u}, \mathbf{w}, \boldsymbol{\phi})+4 v_{r}(\mathbf{w}, \boldsymbol{\phi}) \\
& \quad=d(\mathbf{u}, \phi)+\left\langle\mathbf{f}_{2}, \phi\right\rangle \quad \forall \phi \in \mathbf{H}_{0}^{1}(\Omega) \\
& (\mathbf{u}(0), \mathbf{w}(0))=\left(\mathbf{u}_{0}, \mathbf{w}_{0}\right) .
\end{aligned}
$$

We now give the following proposition on the existence and uniqueness result for (1). They were obtained by $[8,9]$ in a slightly different setting.

Proposition 1. Assume that the given functions $\mathbf{f}_{1}, \mathbf{f}_{2}, \mathbf{g}, \mathbf{q}$, $\mathbf{u}_{0}$, and $\mathbf{w}_{0}$ satisfy $\mathbf{f}_{1}, \mathbf{f}_{2} \in L^{2}\left(0, T ; \mathbf{H}^{-1}(\Omega)\right), \mathbf{g} \in H^{1}(0, T$; $\left.\mathbf{H}^{1 / 2}(\Gamma)\right), \mathbf{q} \in H^{1}\left(0, T ; \mathbf{H}^{1 / 2}(\Gamma)\right), \int_{\Gamma} \mathbf{g} \cdot \mathbf{n} d s=0, \mathbf{u}_{0} \in \mathbf{V}_{\mathbf{g}(\cdot, 0)}$, and $\mathbf{w}_{0} \in \mathbf{H}_{q(\cdot, 0)}^{1}(\Omega)$. Then, problem (15) has at least one solution $(\mathbf{u}, p, \mathbf{w})$ such that $\mathbf{u} \in L^{\infty}\left(0, T ; \mathbf{L}^{2}(\Omega)\right) \cap L^{2}\left(0, T ; \mathbf{V}_{\mathbf{g}}\right), \mathbf{w} \in$ $L^{\infty}\left(0, T ; \mathbf{L}^{2}(\Omega)\right) \cap L^{2}\left(0, T ; \mathbf{H}_{q}^{1}(\Omega)\right)$, and $p \in L^{2}\left(0, T ; L_{0}^{2}(\Omega)\right)$. In two spatial dimensions $(d=2)$, these solutions are unique.
In order to derive the decoupled time-stepping algorithm, we assume $\Omega$ is a convex polyhedral domain, for simplicity, and partition $\Omega$ into a mesh $\mathscr{T}_{h}$ with $\bar{\Omega}=\bigcup_{K \in \mathscr{T}_{h}} K$ so that diameter $(K) \leq h$ and any two closed elements $K_{1}^{h}$ and $K_{2} \epsilon$ $\mathscr{T}_{h}$ either are disjoint or share exactly one face, side, or vertex. Suppose further that $\mathscr{T}_{h}$ is a shape regular and quasiuniform triangulation. On the other hand, we divide the time interval $[0, T]$ into $N$ subintervals $\left[t_{n}, t_{n+1}\right](n=0,1,2, \ldots, N-1)$, satisfying

$$
0<t_{1}<t_{1}<t_{2}<\cdots<t_{N-1}<t_{N}=T .
$$

Let $\Delta t:=t_{n}-t_{n-1}$ be the time step. We introduce the finite element spaces $\mathbb{X}_{h} \subset H^{1}(\Omega)$ and $\mathbb{Q}_{h} \subset L^{2}(\Omega)$ which are divstable: there exists a constant $\beta>0$, independent of $h$, such that

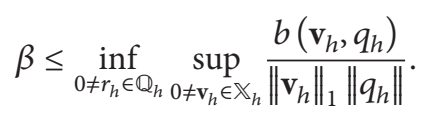

Let $\mathbb{Y}_{h} \subset H^{1}(\Omega)$ be another finite element space and let $\mathbf{g}_{h}$ and $\mathbf{q}_{h}$ be approximations of $\mathbf{g}$ and $\mathbf{q}$, respectively, such that there exist $\mathbf{u}_{h} \in \mathbb{X}_{h}$ and $\mathbf{w}_{h} \in \mathbb{Y}_{h}$ satisfying $\left.\mathbf{u}_{h}\right|_{\Gamma}=\mathbf{g}_{h}$ and $\left.\mathbf{w}_{h}\right|_{\Gamma}=\mathbf{q}_{h}$. We then define $\mathbf{X}_{h, g_{h}}(\Omega):=\mathbb{X}_{h} \cap \mathbf{H}_{g_{h}}^{1}(\Omega), \mathbf{Y}_{h, q_{h}}(\Omega):=\mathbb{V}_{h} \cap$ $\mathbf{H}_{q_{h}}^{1}(\Omega)$, and $Q_{h}:=\mathbb{Q}_{h} \cap L_{0}^{2}(\Omega)$.

We make the following assumptions on the finite dimensional subspaces $\mathbb{X}_{h}, \mathbb{Y}_{h}$, and $\mathbb{Q}_{h}$.

Assumption A1. We have the approximation properties: there exist an integer $k$ and a constant $C$, independent of $h, \mathbf{v}, \mathbf{w}$, and $r$, such that

$$
\begin{array}{r}
\inf _{\mathbf{v}_{h} \in \mathbb{X}_{h}}\left[\left\|\mathbf{v}-\mathbf{v}_{h}\right\|+h\left\|\nabla\left(\mathbf{v}-\mathbf{v}_{h}\right)\right\|\right] \leq C h^{\ell+1}\|\mathbf{v}\|_{\ell+1} \\
\forall \mathbf{v} \in \mathbf{H}^{\ell+1}(\Omega), 1 \leq \ell \leq k, \\
\inf _{\mathbf{w}_{h} \in \mathbb{Y}_{h}}\left[\left\|\mathbf{w}-\mathbf{w}_{h}\right\|+h\left\|\nabla\left(\mathbf{w}-\mathbf{w}_{h}\right)\right\|\right] \leq C h^{\ell+1}\|\mathbf{w}\|_{\ell+1} \\
\forall \mathbf{w} \in \mathbf{H}^{\ell+1}(\Omega), 1 \leq \ell \leq k, \\
\inf _{r_{h} \in Q_{h}}\left\|r-r_{h}\right\| \leq C h^{\ell}\|r\|_{\ell} \\
\forall r \in H^{\ell}(\Omega) .
\end{array}
$$

Assumption A2. For any integers $l$ and $m(0 \leq l \leq m \leq 1)$ and any real numbers $p$ and $q(1 \leq p \leq q \leq \infty)$ it holds that

$$
\left\|\psi_{h}\right\|_{m, q} \leq c h^{l-m+d(1 / q-1 / p)}\left\|\psi_{h}\right\|_{l, p} \quad \forall \psi_{h} \in \mathbb{X}_{h} .
$$

There are many conforming finite element spaces satisfying Assumptions A1 and A2. One may choose, for example, the Taylor-Hood element pair for the velocity and pressure (i.e., piecewise quadratic polynomial for velocity and piecewise linear polynomial for pressure) and piecewise quadratic polynomials for the microrotation vector. Then, Hypotheses $\mathrm{A} 1$ and $\mathrm{A} 2$ hold with $k=2$.

We also cite a discrete Grönwall lemma which is useful in our analysis as follows. 
Lemma 2 (discrete Grönwall lemma [31]). Let $d, \Delta t,\left\{a_{n}\right\}_{n \geq 0}$, $\left\{b_{n}\right\}_{n \geq 0},\left\{c_{n}\right\}_{n \geq 0}$, and $\left\{d_{n}\right\}_{n \geq 0}$ be nonnegative numbers such that

$$
a_{m}+\Delta t \sum_{n=1}^{m} b_{n} \leq \Delta t \sum_{n=0}^{m-1} a_{n} d_{n}+\Delta t \sum_{n=0}^{m-1} c_{n}+d, \quad m \geq 1
$$

Then one has

$$
a_{m}+\Delta t \sum_{n=1}^{m} b_{n} \leq \exp \left(\Delta t \sum_{n=0}^{m-1} d_{n}\right)\left(\Delta t \sum_{n=0}^{m-1} c_{n}+d\right)
$$

$m \geq 1$.

\section{Error Analysis of the Decoupled Time-Stepping Scheme}

In this section, we present the decoupled time-stepping algorithm for the micropolar fluid model and derive error estimates. System (15) is discretized by Crank-Nicholson scheme in time and Galerkin finite element in space. The time discretization combines an implicit treatment of the second derivative terms, a semi-implicit second-order extrapolation for the nonlinear convective terms and explicit treatment of the microrotation vector coupling term in the Navier-Stokes equations.

Let $\left[t_{n}, t_{n+1}\right] \subset \mathbb{R}^{+}$denote a typical time subinterval and let $\phi^{n}(\cdot)$ be a given algorithmic approximation to $\phi\left(\cdot, t_{n}\right)$. Let $\mathscr{D}\left(\phi^{n+1}\right)$ denote the difference operator

$$
\mathscr{D}\left(\phi^{n+1}\right):=\frac{\phi^{n+1}-\phi^{n}}{\Delta t}
$$

and let $\mathscr{I}\left(\phi^{n+1 / 2}\right)$ denote the extrapolation operator $\mathscr{I}\left(\phi^{n+1 / 2}\right):=(3 / 2) \phi^{n}-(1 / 2) \phi^{n-1}$.

Algorithm 3. Given $\left(\mathbf{u}_{h}^{i}, \mathbf{w}_{h}^{i}, p_{h}^{i}\right) \in \mathbf{X}_{h, g_{h}^{i}} \times \mathbf{Y}_{h, q_{h}^{i}} \times Q_{h}, i=0,1$, find $\left\{\left(\mathbf{u}_{h}^{n+1}, \mathbf{w}_{h}^{n+1}, p_{h}^{n+1}\right)\right\} \in \mathbf{X}_{h, g_{h}^{n+1}} \times \mathbf{Y}_{h, q_{h}^{n+1}} \times Q_{h}$ such that

$$
\begin{aligned}
& \left(\mathscr{D}\left(\mathbf{u}_{h}^{n+1}\right), \mathbf{v}_{h}\right)+a_{1}\left(\mathbf{u}_{h}^{n+1 / 2}, \mathbf{v}_{h}\right) \\
& +c\left(\mathscr{I}\left(\mathbf{u}_{h}^{n+1 / 2}\right), \mathbf{u}_{h}^{n+1 / 2}, \mathbf{v}_{h}\right) \\
& +b\left(\mathbf{v}_{h}, p_{h}^{n+1 / 2}\right)=d\left(\mathscr{I}\left(\mathbf{w}_{h}^{n+1 / 2}\right), \mathbf{v}_{h}\right) \\
& \quad+\left\langle\mathbf{f}_{1}\left(t_{n+1 / 2}\right), \mathbf{v}_{h}\right\rangle \quad \forall \mathbf{v}_{h} \in \mathbf{X}_{h}, \\
& b\left(\mathbf{u}_{h}^{n+1 / 2}, r_{h}\right)=0 \quad \forall r_{h} \in Q_{h}, \\
& \left(\mathscr{D}\left(\mathbf{w}_{h}^{n+1}\right), \phi_{h}\right)+a_{2}\left(\mathbf{w}_{h}^{n+1 / 2}, \phi_{h}\right) \\
& +c\left(\mathscr{I}\left(\mathbf{u}_{h}^{n+1 / 2}\right), \mathbf{w}_{h}^{n+1 / 2}, \boldsymbol{\phi}_{h}\right) \\
& +4 v_{r}\left(\mathbf{w}_{h}^{n+1 / 2}, \boldsymbol{\phi}_{h}\right)=d\left(\mathbf{u}_{h}^{n+1 / 2}, \boldsymbol{\phi}_{h}\right) \\
& +\left\langle\mathbf{f}_{2}\left(t_{n+1 / 2}\right), \phi_{h}\right\rangle \quad \forall \phi_{h} \in \mathbf{Y}_{h},
\end{aligned}
$$

for $n=1,2, \ldots, N-1$, where $\mathbf{u}_{h}^{n+1 / 2}, \mathbf{w}_{h}^{n+1}$, and $p_{h}^{n+1 / 2}$ are the intermediate variables defined by $\mathbf{u}_{h}^{n+1 / 2}:=(1 / 2) \mathbf{u}_{h}^{n+1}+$ $(1 / 2) \mathbf{u}_{h}^{n}, \mathbf{w}_{h}^{n+1 / 2}:=(1 / 2) \mathbf{w}_{h}^{n+1}+(1 / 2) \mathbf{w}_{h}^{n}$, and $p_{h}^{n+1 / 2}:=$ $(1 / 2) p_{h}^{n+1}+(1 / 2) p_{h}^{n}$, respectively.

3.1. Error Analysis of Decoupled Scheme. In this section, we will derive error estimates of the decoupled Crank-Nicolson scheme proposed above. For simplicity, we will assume the boundary data is independent of time in the subsequent analysis.

Let us define two projections, namely, Stokes and generalized Ritz projections, as follows: given $(\mathbf{u}, p) \in \mathbf{H}^{1}(\Omega) \times L_{0}^{2}(\Omega)$ and $\mathbf{w} \in \mathbf{H}^{1}(\Omega)$, we define the Stokes projection $\left(P_{h}^{s} \mathbf{u}, P_{h}^{s} p\right) \in$ $\mathbf{X}_{h, g_{h}} \times Q_{h}$ as the solution of the problem

$$
\begin{aligned}
a_{1}\left(\left(\mathbf{u}-P_{h}^{s} \mathbf{u}\right), \mathbf{v}_{h}\right)+b\left(\mathbf{v}_{h},\left(p-P_{h}^{s} p\right)\right)=0 & \\
& \forall \mathbf{v}_{h} \in \mathbf{X}_{h}, \\
b\left(\mathbf{u}-P_{h}^{s} \mathbf{u}, r_{h}\right)=0 & \forall r_{h} \in Q_{h}
\end{aligned}
$$

and the generalized Ritz projection $P_{h}^{r} \mathbf{w} \in \mathbf{Y}_{h, q_{h}}$ as the solution of the problem

$$
a_{2}\left(\left(\mathbf{w}-P_{h}^{r} \mathbf{w}\right), \psi_{h}\right)=0 \quad \forall \psi_{h} \in \mathbf{Y}_{h} .
$$

Using the $H^{2}$-regularity property of the Stokes and Ritz operators in smooth domains and a duality argument, we can show the following approximation properties hold:

$$
\begin{gathered}
\left\|\mathbf{u}-P_{h}^{s} \mathbf{u}\right\|_{1}+\left\|p-P_{h}^{s} p\right\| \leq \operatorname{ch}^{k}\left(\|\mathbf{u}\|_{k+1}+\|p\|_{k}\right) \\
\left\|\mathbf{w}-P_{h}^{r} \mathbf{w}\right\|_{1} \leq c h^{k}\|\mathbf{w}\|_{k+1} .
\end{gathered}
$$

Moreover, these approximation properties together with (11)(12) yield

$$
\begin{aligned}
& \left\|P_{h}^{s} \mathbf{u}\right\|_{\infty}+\left\|P_{h}^{s} \mathbf{u}\right\|_{1,3} \leq c\left(\|\mathbf{u}\|_{2}+\|p\|_{1}\right), \\
& \left\|P_{h}^{r} \mathbf{w}\right\|_{\infty}+\left\|P_{h}^{r} \mathbf{w}\right\|_{1,3} \leq c\|\mathbf{w}\|_{2} .
\end{aligned}
$$

Moreover, under certain smoothness assumptions on $\phi$, we have by Taylor expansion with integral remainder

$$
\begin{aligned}
& \left\|\phi^{n+1 / 2}-\phi\left(t_{n+1 / 2}\right)\right\|_{k}^{2} \leq \frac{(\Delta t)^{3}}{48} \int_{t_{n}}^{t_{n+1}}\left\|\partial_{t}^{2} \phi\right\|_{k}^{2} d t, \\
& \left\|\partial_{t} \phi\left(t_{n+1 / 2}\right)-\mathscr{D}\left(\phi\left(t_{n+1}\right)\right)\right\|^{2} \\
& \quad \leq \frac{(\Delta t)^{3}}{1280} \int_{t_{n}}^{t_{n+1}}\left\|\partial_{t}^{3} \phi(t)\right\|^{2} d t, \\
& \left\|\mathscr{I}\left(\phi\left(t_{n+1 / 2}\right)\right)-\phi\left(t_{n+1 / 2}\right)\right\|_{H^{k}} \\
& \quad \leq c(\Delta t)^{3 / 2}\left\|\partial_{t}^{2} \phi(t)\right\|_{L^{2}\left(t_{n-1}, t_{n+1 / 2} ; H^{k}\right)} .
\end{aligned}
$$

Under the above-mentioned assumptions, we can obtain the following error estimate for the velocity. 
Theorem 4. Suppose that Assumptions A1 and A2 hold with a positive number $h_{0}$ and a positive integer $k$ that the solution $(\mathbf{u}, \mathbf{w}, p)$ of (15) satisfies

$$
\begin{aligned}
\mathbf{u} \in \mathscr{C}\left([0, T] ; \mathbf{V}_{g}\right) \cap H^{1}\left(0, T ; \mathbf{H}^{k+1}(\Omega)\right) \\
\cap H^{3}\left(0, T ; \mathbf{L}^{2}(\Omega)\right), \\
\mathbf{w} \in \mathscr{C}\left([0, T] ; \mathbf{H}_{q}^{1}\right) \cap H^{1}\left(0, T ; \mathbf{H}^{k+1}(\Omega)\right) \\
\quad \cap H^{3}\left(0, T ; \mathbf{L}^{2}(\Omega)\right), \\
p \in \mathscr{C}\left([0, T] ; L_{0}^{2}(\Omega) \cap H^{k}(\Omega)\right)
\end{aligned}
$$

and that the initial conditions $\left(\mathbf{u}_{h}^{i}, \mathbf{w}_{h}^{i}\right), i=0,1$ satisfy $\sum_{i=0}^{1}\left\|\left(\mathbf{u}_{h}^{i}, \mathbf{w}_{h}^{i}\right)-\left(\mathbf{u}\left(t_{i}\right), \mathbf{w}\left(t_{i}\right)\right)\right\| \leq c h^{k}$. Then, for any $h \in\left(0, h_{0}\right]$ the approximate solutions $\left(\mathbf{u}_{h}, \mathbf{w}_{h}\right)$ of (23) satisfy the following error estimates:

$$
\begin{aligned}
&\left\|\mathbf{u}-\mathbf{u}_{h}\right\|_{l^{\infty}\left(L^{2}(\Omega)\right) \cap l^{2}\left(\mathbf{H}^{1}(\Omega)\right)} \leq C\left(\Delta t^{2}+h^{k}\right), \\
&\left\|\mathbf{w}-\mathbf{w}_{h}\right\|_{l^{\infty}\left(L^{2}(\Omega)\right) \cap l^{2}\left(\mathbf{H}^{1}(\Omega)\right)} \leq C\left(\Delta t^{2}+h^{k}\right)
\end{aligned}
$$

for some constant $C$ independent of the mesh size $h$ and time step $\Delta t$.

Proof. Let us denote the Stokes projection $\left(P_{h}^{s} \mathbf{u}\left(t_{n}\right), P_{h}^{s} p\left(t_{n}\right)\right)$ and generalized Ritz projection $P_{h}^{r} \mathbf{w}\left(t_{n}\right)$ by $\left(\overline{\mathbf{u}}\left(t_{n}\right), \bar{p}\left(t_{n}\right)\right)$ and $\overline{\mathbf{w}}\left(t_{n}\right)$, respectively, for convenience. Moreover, let $\left(\mathbf{e}_{1 h}^{n}\right.$, $\left.e_{2 h}^{n}, \mathbf{e}_{3 h}^{n}\right)$ be the errors defined by

$$
\begin{aligned}
\mathbf{e}_{1 h}^{n} & :=\mathbf{u}_{h}^{n}-\overline{\mathbf{u}}\left(t_{n}\right), \\
e_{2 h}^{n} & :=p_{h}^{n}-\bar{p}\left(t_{n}\right), \\
\mathbf{e}_{3 h}^{n} & :=\mathbf{w}_{h}^{n}-\overline{\mathbf{w}}\left(t_{n}\right) .
\end{aligned}
$$

Then by the approximation properties (26)-(27), we need to only estimate $\mathbf{e}_{1 h}^{n}$ and $e_{2 h}^{n}$ in order to furnish the desired error estimates. To this end, we first subtract (15) from (23) and obtain

$$
\begin{aligned}
& \left(\mathscr{D}\left(\mathbf{u}_{h}^{n+1}\right)-\partial_{t} \mathbf{u}\left(t_{n+1 / 2}\right), \mathbf{v}_{h}\right)+a_{1}\left(\mathbf{u}_{h}^{n+1 / 2}, \mathbf{v}_{h}\right) \\
& \quad+b\left(\mathbf{v}_{h}, p_{h}^{n+1 / 2}\right)=\left\langle\aleph_{h}^{n}, \mathbf{v}_{h}\right\rangle+a_{1}\left(\mathbf{u}\left(t_{n+1 / 2}\right), \mathbf{v}_{h}\right) \\
& \quad+b\left(\mathbf{v}_{h}, p\left(t_{n+1 / 2}\right)\right) \quad \forall \mathbf{v}_{h} \in \mathbf{X}_{h}, \\
& b\left(\mathbf{u}_{h}^{n+1 / 2}, r_{h}\right)=b\left(\mathbf{u}\left(t_{n+1 / 2}\right), r_{h}\right) \quad \forall r_{h} \in Q_{h}, \\
& \left(\mathscr{D}\left(\mathbf{w}_{h}^{n+1}\right)-\partial_{t} \mathbf{w}\left(t_{n+1 / 2}\right), \phi_{h}\right)+a_{2}\left(\mathbf{w}_{h}^{n+1 / 2}, \phi_{h}\right) \\
& \quad+4 v_{r}\left(\mathbf{w}_{h}^{n+1 / 2}-\mathbf{w}\left(t_{n+1 / 2}\right), \phi_{h}\right) \\
& \quad=a_{2}\left(\mathbf{w}\left(t_{n+1 / 2}\right), \phi_{h}\right)+\left\langle\widehat{\aleph}_{h}^{n}, \phi_{h}\right\rangle \quad \forall \phi_{h} \in \mathbf{Y}_{h},
\end{aligned}
$$

at each time step $n$, where $\aleph_{h}^{n}$ and $\widehat{\aleph}_{h}^{n}$ are defined by

$$
\begin{aligned}
\left\langle\aleph_{h}^{n}, \mathbf{v}_{h}\right\rangle:= & c\left(\mathbf{u}\left(t_{n+1 / 2}\right), \mathbf{u}\left(t_{n+1 / 2}\right), \mathbf{v}_{h}\right) \\
& -c\left(\mathscr{I}\left(\mathbf{u}_{h}^{n+1 / 2}\right), \mathbf{u}_{h}^{n+1 / 2}, \mathbf{v}_{h}\right) \\
& +d\left(\mathscr{I}\left(\mathbf{w}_{h}^{n+1 / 2}\right)-\mathbf{w}\left(t_{n+1 / 2}\right), \mathbf{v}_{h}\right), \\
\left\langle\widehat{\aleph}_{h}^{n}, \boldsymbol{\phi}_{h}\right\rangle:= & c\left(\mathbf{u}\left(t_{n+1 / 2}\right), \mathbf{w}\left(t_{n+1 / 2}\right), \boldsymbol{\phi}_{h}\right) \\
& -c\left(\mathscr{I}\left(\mathbf{u}_{h}^{n+1 / 2}\right), \mathbf{w}_{h}^{n+1 / 2}, \boldsymbol{\phi}_{h}\right) \\
& +d\left(\mathbf{u}_{h}^{n+1 / 2}-\mathbf{u}\left(t_{n+1 / 2}\right), \boldsymbol{\phi}\right) .
\end{aligned}
$$

Using the definition of Stokes and generalized Ritz projections, we obtain the basic error equations of the method

$$
\begin{gathered}
\left(\mathscr{D}\left(\mathbf{e}_{1 h}^{n+1}\right), \mathbf{v}_{h}\right)+a_{1}\left(\mathbf{e}_{1 h}^{n+1 / 2}, \mathbf{v}_{h}\right)+b\left(\mathbf{v}_{h}, e_{2 h}^{n+1 / 2}\right) \\
=\left\langle\aleph_{h}^{n}, \mathbf{v}_{h}\right\rangle+\left(\partial_{t} \mathbf{u}\left(t_{n+1 / 2}\right)-\mathscr{D} \overline{\mathbf{u}}\left(t_{n+1}\right), \mathbf{v}_{h}\right) \\
\forall \mathbf{v}_{h} \in \mathbf{X}_{h}, \\
b\left(\mathbf{e}_{1 h}^{n+1 / 2}, r_{h}\right)=0 \quad \forall r_{h} \in Q_{h}, \\
\left(\mathscr{D}\left(\mathbf{e}_{3 h}^{n+1}\right), \phi_{h}\right)+a_{2}\left(\mathbf{e}_{3 h}^{n+1 / 2}, \phi_{h}\right)+4 v_{r}\left(\mathbf{e}_{3 h}^{n+1 / 2}, \boldsymbol{\phi}_{h}\right) \\
=\left(\partial_{t} \mathbf{w}\left(t_{n+1 / 2}\right)-\mathscr{D} \overline{\mathbf{w}}\left(t_{n+1}\right), \phi_{h}\right)+\left\langle\widehat{\aleph}_{h}^{n}, \phi_{h}\right\rangle \\
+4 v_{r}\left(\mathbf{w}\left(t_{n+1 / 2}\right)-\overline{\mathbf{w}}\left(t_{n+1 / 2}\right), \phi_{h}\right) \quad \forall \phi_{h} \in \mathbf{Y}_{h} .
\end{gathered}
$$

We next split the nonlinear terms $\left\langle\aleph_{h}^{n}, \mathbf{v}_{h}\right\rangle$ and $\left\langle\widehat{\aleph}_{h}^{n}, \phi_{h}\right\rangle$ on the right-hand side of (37) into several terms as follows:

$$
\begin{aligned}
& \left\langle\aleph_{h}^{n}, \mathbf{v}_{h}\right\rangle=c\left(\mathbf{u}\left(t_{n+1 / 2}\right), \mathbf{u}\left(t_{n+1 / 2}\right)-\overline{\mathbf{u}}\left(t_{n+1 / 2}\right), \mathbf{v}_{h}\right) \\
& \quad+c\left(\mathbf{u}\left(t_{n+1 / 2}\right)-\mathscr{I}\left(\mathbf{u}\left(t_{n+1 / 2}\right)\right), \overline{\mathbf{u}}\left(t_{n+1 / 2}\right), \mathbf{v}_{h}\right) \\
& \quad+c\left(\mathscr{I}\left(\mathbf{u}\left(t_{n+1 / 2}\right)\right)\right. \\
& \left.\quad-\mathscr{I}\left(\overline{\mathbf{u}}\left(t_{n+1 / 2}\right)\right), \overline{\mathbf{u}}\left(t_{n+1 / 2}\right), \mathbf{v}_{h}\right) \\
& \quad-c\left(\mathscr{I}\left(\mathbf{e}_{1 h}^{n+1 / 2}\right), \overline{\mathbf{u}}\left(t_{n+1 / 2}\right), \mathbf{v}_{h}\right) \\
& \quad-c\left(\mathscr{I}\left(\overline{\mathbf{u}}\left(t_{n+1 / 2}\right)\right), \mathbf{e}_{1 h}^{n+1 / 2}, \mathbf{v}_{h}\right) \\
& \quad-c\left(\mathscr{I}\left(\mathbf{e}_{1 h}^{n+1 / 2}\right), \mathbf{e}_{1 h}^{n+1 / 2}, \mathbf{v}_{h}\right)+d\left(\mathscr{I}\left(\overline{\mathbf{w}}\left(t_{n+1 / 2}\right)\right)\right. \\
& \left.\quad-\mathbf{w}\left(t_{n+1 / 2}\right), \mathbf{v}_{h}\right)+d\left(\mathscr{I}\left(\mathbf{e}_{3 h}^{n+1 / 2}\right), \mathbf{v}_{h}\right) \\
& \quad=\sum_{i=1}^{8}\left\langle\aleph_{i}^{n}, \mathbf{v}_{h}\right\rangle, \\
& \left\langle\widehat{\aleph}_{h}^{n}, \phi_{h}\right\rangle=c\left(\mathbf{u}\left(t_{n+1 / 2}\right), \mathbf{w}\left(t_{n+1 / 2}\right)-\overline{\mathbf{w}}\left(t_{n+1 / 2}\right), \phi_{h}\right) \\
& +c\left(\mathbf{u}\left(t_{n+1 / 2}\right)-\mathscr{I}\left(\mathbf{u}\left(t_{n+1 / 2}\right)\right), \overline{\mathbf{w}}\left(t_{n+1 / 2}\right), \phi_{h}\right) \\
& +c\left(\mathscr{I}\left(\mathbf{u}\left(t_{n+1 / 2}\right)\right)\right.
\end{aligned}
$$




$$
\begin{aligned}
& \left.-\mathscr{I}\left(\overline{\mathbf{u}}\left(t_{n+1 / 2}\right)\right), \overline{\mathbf{w}}\left(t_{n+1 / 2}\right), \boldsymbol{\phi}_{h}\right) \\
& -c\left(\mathscr{I}\left(\mathbf{e}_{1 h}^{n+1 / 2}\right), \overline{\mathbf{w}}\left(t_{n+1 / 2}\right), \boldsymbol{\phi}_{h}\right) \\
& -c\left(\mathscr{I}\left(\overline{\mathbf{u}}\left(t_{n+1 / 2}\right)\right), e_{3 h}^{n+1 / 2}, \boldsymbol{\phi}_{h}\right) \\
& -c\left(\mathscr{I}\left(\mathbf{e}_{1 h}^{n+1 / 2}\right), e_{3 h}^{n+1 / 2}, \boldsymbol{\phi}_{h}\right)+d\left(\mathbf{e}_{1 h}^{n+1 / 2}, \boldsymbol{\phi}_{h}\right) \\
& +d\left(\overline{\mathbf{u}}\left(t_{n+1 / 2}\right)-\mathbf{u}\left(t_{n+1 / 2}\right), \boldsymbol{\phi}_{h}\right)=: \sum_{i=1}^{8}\left\langle\widehat{\aleph}_{i}^{n}, \phi_{h}\right\rangle .
\end{aligned}
$$

Notice $\left\langle\aleph_{5}^{n}, \mathbf{e}_{1 h}^{n+1 / 2}\right\rangle=\left\langle\aleph_{6}^{n}, \mathbf{e}_{1 h}^{n+1 / 2}\right\rangle=\left\langle\widehat{\aleph}_{5}^{n}, \mathbf{e}_{3 h}^{n+1 / 2}\right\rangle=\left\langle\widehat{\aleph}_{6}^{n}\right.$, $\left.\mathbf{e}_{3 h}^{n+1 / 2}\right\rangle=0$ due to skew-symmetry of trilinear form $c(\cdot, \cdot, \cdot)$. Therefore, setting $\left(\mathbf{v}_{h}, \phi_{h}\right)=\left(\Delta t \mathbf{e}_{1 h}^{n+1 / 2}, \Delta t \mathbf{e}_{3 h}^{n+1 / 2}\right)$ into (37) we can write it as

$$
\begin{aligned}
& \left\|\mathbf{e}_{1 h}^{n+1}\right\|^{2}-\left\|\mathbf{e}_{1 h}^{n}\right\|^{2}+\Delta t\left(\nu+v_{r}\right)\left\|\nabla \mathbf{e}_{1 h}^{n+1 / 2}\right\|^{2} \\
& =\Delta t\left(\partial_{t} \mathbf{u}\left(t_{n+1 / 2}\right)-\mathscr{D} \overline{\mathbf{u}}\left(t_{n+1}\right), \mathbf{e}_{1 h}^{n+1 / 2}\right) \\
& +\Delta t \sum_{i=1}^{4}\left\langle\aleph_{i}^{n}, \mathbf{e}_{1 h}^{n+1 / 2}\right\rangle+\Delta t \sum_{i=7}^{8}\left\langle\aleph_{i}^{n}, \mathbf{e}_{1 h}^{n+1 / 2}\right\rangle, \\
& \left\|\mathbf{e}_{3 h}^{n+1}\right\|^{2}-\left\|\mathbf{e}_{3 h}^{n}\right\|^{2}+\left(c_{a}+c_{d}\right) \Delta t\left\|\nabla \mathbf{e}_{3 h}^{n+1 / 2}\right\|^{2} \\
& \quad+4 v_{r} \Delta t\left\|\mathbf{e}_{3 h}^{n+1 / 2}\right\|^{2}+\left(c_{0}+c_{d}-c_{a}\right) \Delta t\left\|\nabla \cdot \mathbf{e}_{3 h}^{n+1 / 2}\right\|^{2} \\
& \quad=\Delta t\left(\partial_{t} \mathbf{w}\left(t_{n+1 / 2}\right)-\mathscr{D}\left(\overline{\mathbf{w}}^{n+1}\right), \mathbf{e}_{3 h}^{n+1 / 2}\right) \\
& +4 v_{r} \Delta t\left(\mathbf{w}\left(t_{n+1 / 2}\right)-\overline{\mathbf{w}}\left(t_{n+1 / 2}\right), \mathbf{e}_{3 h}^{n+1 / 2}\right) \\
& \quad+\Delta t \sum_{i=1}^{4}\left\langle\widehat{\aleph}_{h}^{n}, \mathbf{e}_{3 h}^{n+1 / 2}\right\rangle+\Delta t \sum_{i=7}^{8}\left\langle\widehat{\aleph}_{h}^{n}, \mathbf{e}_{3 h}^{n+1 / 2}\right\rangle .
\end{aligned}
$$

We proceed to bound each term on the right-hand side of (39) and absorb like-terms into the left-hand side. We begin with the first terms on the right-hand side of $(39)_{1}$ and $(39)_{2}$. Notice that by triangle inequality

$$
\begin{aligned}
\left\|\partial_{t} \mathbf{u}\left(t_{n+1 / 2}\right)-\mathscr{D} \overline{\mathbf{u}}\left(t_{n+1}\right)\right\| & \\
\leq & \left\|\partial_{t} \mathbf{u}\left(t_{n+1 / 2}\right)-\mathscr{D} \mathbf{u}\left(t_{n+1}\right)\right\| \\
& \quad\left\|\mathscr{D} \mathbf{u}\left(t_{n+1}\right)-\mathscr{D} \overline{\mathbf{u}}\left(t_{n+1}\right)\right\| .
\end{aligned}
$$

It is easy to verify, by Cauchy-Schwarz inequality, that

$$
\begin{aligned}
& \left\|\mathscr{D} \mathbf{u}\left(t_{n+1}\right)-\mathscr{D} \overline{\mathbf{u}}\left(t_{n+1}\right)\right\|=\frac{1}{\Delta t}\left\|\int_{t_{n}}^{t_{n+1}}\left(P_{h}^{s}-I\right) \partial_{t} \mathbf{u} d t\right\| \\
& \quad \leq \frac{1}{\sqrt{\Delta t}}\left\|\left(P_{h}^{s}-I\right) \partial_{t} \mathbf{u}\right\|_{L^{2}\left(t_{n}, t_{n+1} ; \mathbf{L}(\Omega)\right)} .
\end{aligned}
$$

Combining this with Stokes projection approximation property (26) and estimate (30), we obtain

$$
\begin{aligned}
& \left\|\partial_{t} \mathbf{u}\left(t_{n+1 / 2}\right)-\mathscr{D} \overline{\mathbf{u}}\left(t_{n+1}\right)\right\| \\
& \quad \leq c\left\{(\Delta t)^{3 / 2}\left\|\partial_{t}^{3} \mathbf{u}\right\|_{L^{2}\left(t_{n}, t_{n+1} ; \mathbf{L}^{2}(\Omega)\right)}\right. \\
& \left.+\frac{h^{k}}{\sqrt{\Delta t}}\left\|\left(\partial_{t} \mathbf{u}, \partial_{t} p\right)\right\|_{L^{2}\left(t_{n}, t_{n+1} ; \mathbf{H}^{k+1}(\Omega) \times H^{k}(\Omega)\right)}\right\} .
\end{aligned}
$$

In the same way, we can show

$$
\begin{aligned}
& \left\|\partial_{t} \mathbf{w}\left(t_{n+1 / 2}\right)-\mathscr{D} \overline{\mathbf{w}}\left(t_{n+1}\right)\right\| \\
& \quad \leq c\left\{(\Delta t)^{3 / 2}\left\|\partial_{t}^{3} \mathbf{w}\right\|_{L^{2}\left(t_{n}, t_{n+1} ; \mathbf{L}^{2}(\Omega)\right)}\right. \\
& \left.+\frac{h^{k}}{\sqrt{\Delta t}}\left\|\partial_{t} \mathbf{w}\right\|_{L^{2}\left(t_{n}, t_{n+1} ; \mathbf{H}^{k+1}(\Omega)\right)}\right\} .
\end{aligned}
$$

By Cauchy-Schwarz inequality and (42) and (43), we have

$$
\begin{aligned}
& \left(\partial_{t} \mathbf{u}\left(t_{n+1 / 2}\right)-\mathscr{D} \overline{\mathbf{u}}\left(t_{n+1}\right), \mathbf{e}_{1 h}^{n+1 / 2}\right) \\
& \quad \leq C\left\{(\Delta t)^{3 / 2}\left\|\partial_{t}^{3} \mathbf{u}\right\|_{L^{2}\left(t_{n}, t_{n+1} ; \mathbf{L}^{2}(\Omega)\right)}\right. \\
& \left.\quad+\frac{h^{k}}{\sqrt{\Delta t}}\left\|\left(\partial_{t} \mathbf{u}, \partial_{t} p\right)\right\|_{L^{2}\left(t_{n}, t_{n+1} ; \mathbf{H}^{k+1}(\Omega) \times H^{k}(\Omega)\right)}\right\}\left\|\mathbf{e}_{1 h}^{n+1 / 2}\right\|, \\
& \left(\partial_{t} \mathbf{w}\left(t_{n+1 / 2}\right)-\mathscr{D} \overline{\mathbf{w}}\left(t_{n+1}\right), \mathbf{e}_{3 h}^{n+1 / 2}\right) \\
& \quad \leq C\left\{(\Delta t)^{3 / 2}\left\|\partial_{t}^{3} \mathbf{w}\right\|_{L^{2}\left(t_{n}, t_{n+1} ; \mathbf{L}^{2}(\Omega)\right)}\right. \\
& \left.\quad+\frac{h^{k}}{\sqrt{\Delta t}}\left\|\partial_{t} \mathbf{w}\right\|_{L^{2}\left(t_{n}, t_{n+1} ; \mathbf{H}^{k+1}(\Omega)\right)}\right\}\left\|\mathbf{e}_{3 h}^{n+1 / 2}\right\| .
\end{aligned}
$$

Using Hölder's inequality, Sobolev inequality, and (26) and (31), we obtain

$$
\begin{aligned}
& \left|\left\langle\aleph_{1}^{n}, \mathbf{e}_{1 h}^{n+1 / 2}\right\rangle\right| \leq c^{*}\left\|\mathbf{u}\left(t_{n+1 / 2}\right)\right\|_{1} \\
& \cdot\left\|\mathbf{u}\left(t_{n+1 / 2}\right)-\overline{\mathbf{u}}\left(t_{n+1 / 2}\right)\right\|_{1}\left\|\mathbf{e}_{1 h}^{n+1 / 2}\right\|_{1} \\
& \leq c^{*} h^{k}\|(\mathbf{u}, p)\|_{\mathscr{C}\left(\left[t_{n}, t_{n+1}\right] ; \mathbf{H}^{k+1}(\Omega) \times \mathbf{H}^{k}(\Omega)\right)}\left\|\mathbf{e}_{1 h}^{n+1 / 2}\right\|, \\
& \left|\left\langle\aleph_{2}^{n}, \mathbf{e}_{1 h}^{n+1 / 2}\right\rangle\right| \leq c^{*}\left\|\mathbf{u}\left(t_{n+1 / 2}\right)-\mathscr{I}\left(\mathbf{u}\left(t_{n+1 / 2}\right)\right)\right\| \\
& \cdot\left(\left\|\nabla \overline{\mathbf{u}}\left(t_{n+1 / 2}\right)\right\|_{L^{3}(\Omega)}+\left\|\overline{\mathbf{u}}\left(t_{n+1 / 2}\right)\right\|_{\infty}\right)\left\|\mathbf{e}_{1 h}^{n+1 / 2}\right\|_{1} \\
& \leq c^{*}(\Delta t)^{3 / 2}\left\|\partial_{t}^{2} \mathbf{u}\right\|_{L^{2}\left(t_{n}, t_{n+1}:^{2}(\Omega)\right)}\left\|\mathbf{e}_{1 h}^{n+1 / 2}\right\|_{1},
\end{aligned}
$$




$$
\begin{aligned}
& \left|\left\langle\aleph_{3}^{n}, \mathbf{e}_{1 h}^{n+1 / 2}\right\rangle\right| \leq c^{*}\left\|\mathscr{I}\left(\mathbf{u}\left(t_{n+1 / 2}\right)\right)-\mathscr{I}\left(\overline{\mathbf{u}}\left(t_{n+1 / 2}\right)\right)\right\|_{1} \\
& \cdot\left(\left\|\overline{\mathbf{u}}\left(t_{n+1 / 2}\right)\right\|_{\infty}+\left\|\nabla \overline{\mathbf{u}}\left(t_{n+1 / 2}\right)\right\|_{L^{3}(\Omega)}\right)\left\|\mathbf{e}_{1 h}^{n+1 / 2}\right\| \\
& \leq c^{*} h^{k}\|(\mathbf{u}, p)\|_{\mathscr{C}\left(\left[t_{n}, t_{n+1}\right] ; \mathbf{H}^{k+1}(\Omega) \times \mathbf{H}^{k}(\Omega)\right)}\left\|\mathbf{e}_{1 h}^{n+1 / 2}\right\|, \\
& \left|\left\langle\aleph_{4}^{n}, \mathbf{e}_{1 h}^{n+1 / 2}\right\rangle\right| \leq c^{*}\left\|\mathscr{I}\left(\mathbf{e}_{1 h}^{n+1 / 2}\right)\right\| \\
& \cdot\left(\left\|\overline{\mathbf{u}}\left(t_{n+1 / 2}\right)\right\|_{\infty}+\left\|\nabla \overline{\mathbf{u}}\left(t_{n+1 / 2}\right)\right\|_{L^{3}(\Omega)}\right)\left\|\mathbf{e}_{1 h}^{n+1 / 2}\right\|_{1} \\
& \leq c^{*}\left(\left\|\mathbf{e}_{1 h}^{n}\right\|+\left\|\mathbf{e}_{1 h}^{n-1}\right\|\right)\left\|\mathbf{e}_{1 h}^{n+1 / 2}\right\|_{1} \cdot
\end{aligned}
$$

We next estimate $\left\langle\aleph_{7}^{n}, \mathbf{e}_{1 h}^{n+1 / 2}\right\rangle$ and $\left\langle\aleph_{8}^{n}, \mathbf{e}_{1 h}^{n+1 / 2}\right\rangle$. To this end, first notice that $\int_{\Omega} \nabla \times \mathbf{w} \cdot \mathbf{v} d \Omega=\int_{\Omega} \mathbf{w} \cdot \nabla \times \mathbf{v} d \Omega$ for $\mathbf{v} \epsilon$ $\mathbf{H}_{0}^{1}(\Omega)$. Therefore, using this identity, Hölder's inequality and (27) and (31) we find that

$$
\begin{gathered}
\left|\left\langle\aleph_{7}^{n}, \mathbf{e}_{1 h}^{n+1 / 2}\right\rangle\right| \leq c\left\|\mathscr{I}\left(\overline{\mathbf{w}}\left(t_{n+1 / 2}\right)\right)-\mathbf{w}\left(t_{n+1 / 2}\right)\right\| \\
\cdot\left\|\mathbf{e}_{1 h}^{n+1 / 2}\right\|_{1} \leq c\left[h^{k}\|\mathbf{w}\|_{\mathscr{C}\left(\left[t_{n-1}, t_{n+1}\right] ; \mathbf{H}^{k+1}(\Omega)\right)}\right. \\
\left.+(\Delta t)^{3 / 2}\left\|\partial_{t}^{2} \mathbf{w}\right\|_{L^{2}\left(t_{n}, t_{n+1 / 2} ; L^{2}(\Omega)\right)}\right]\left\|\mathbf{e}_{1 h}^{n+1 / 2}\right\|_{1} .
\end{gathered}
$$

Arguing similarly we obtain

$$
\begin{aligned}
\left|\left\langle\aleph_{8}^{n}, \mathbf{e}_{1 h}^{n+1 / 2}\right\rangle\right| & =\left|2 v_{r}\left(\mathscr{I}\left(\mathbf{e}_{3 h}^{n+1 / 2}\right), \nabla \times \mathbf{e}_{1 h}^{n+1 / 2}\right)\right| \\
& \leq 2 \sqrt{2} v_{r}\left\|\mathscr{I}\left(\mathbf{e}_{3 h}^{n+1 / 2}\right)\right\|\left\|\nabla \mathbf{e}_{1 h}^{n+1 / 2}\right\| .
\end{aligned}
$$

Thus combining these estimates and using Young's inequality, we have

$$
\begin{aligned}
& \sum_{i=1}^{4}\left|\left\langle\aleph_{i}^{n}, \mathbf{e}_{1 h}^{n+1 / 2}\right\rangle\right|+\sum_{i=7}^{8}\left|\left\langle\aleph_{i}^{n}, \mathbf{e}_{1 h}^{n+1 / 2}\right\rangle\right| \\
& \quad \leq c\left\{h^{2 k}\|(\mathbf{u}, p)\|_{\mathscr{C}\left(\left[t_{n}, t_{n+1}\right] ; \mathbf{H}^{k+1}(\Omega) \times \mathbf{H}^{k}(\Omega)\right)}^{2}\right. \\
& \quad+h^{2 k}\|\mathbf{w}\|_{\mathscr{C}\left(\left[t_{n}, t_{n+1}\right] ; H^{k+1}(\Omega)\right)}^{2} \\
& \quad+(\Delta t)^{3}\left\|\left(\partial_{t}^{2} \mathbf{u}, \partial_{t}^{2} \mathbf{w}\right)\right\|_{L^{2}\left(t_{n}, t_{n+1} ; \mathbf{L}^{2}(\Omega)\right)}^{2}+\left\|\mathbf{e}_{1 h}^{n}\right\|^{2} \\
& \left.\quad+\left\|\mathbf{e}_{1 h}^{n-1}\right\|^{2}+\left\|\mathbf{e}_{3 h}^{n}\right\|^{2}+\left\|\mathbf{e}_{3 h}^{n-1}\right\|^{2}\right\}+\frac{v_{r}}{2}\left\|\nabla \mathbf{e}_{1 h}^{n+1 / 2}\right\|^{2} .
\end{aligned}
$$

We can estimate $\left\langle\widehat{\aleph}_{j}^{n}, \mathrm{e}_{3 h}^{n+1 / 2}\right\rangle, j=1,2,3,4$ similarly using Hölder's inequality, Sobolev inequality, and approximation properties. Therefore, we obtain

$$
\begin{aligned}
& \sum_{i=1}^{4}\left|\left\langle\widehat{\aleph}_{i}^{n}, \mathbf{e}_{3 h}^{n+1 / 2}\right\rangle\right| \leq c\left\{(\Delta t)^{3}\left\|\partial_{t}^{2} \mathbf{u}\right\|_{L^{2}\left(t_{n}, t_{n+1} ; L^{2}(\Omega)\right)}^{2}\right. \\
& +h^{2 k}\|(\mathbf{u}, p)\|_{\mathscr{C}\left(\left[t_{n}, t_{n+1}\right] ; \mathbf{H}^{k+1}(\Omega) \times H^{k}(\Omega)\right)}^{2} \\
& \left.+h^{2 k}\|\mathbf{w}\|_{\mathscr{C}\left(\left[t_{n}, t_{n+1}\right] ; \mathbf{H}^{k+1}(\Omega)\right)}^{2}+\left\|\mathbf{e}_{1 h}^{n}\right\|^{2}+\left\|\mathbf{e}_{1 h}^{n-1}\right\|^{2}\right\} \\
& \quad+\frac{c_{a}+c_{d}}{8}\left\|\nabla \mathbf{e}_{3 h}^{n+1 / 2}\right\|^{2} .
\end{aligned}
$$

In order to estimate terms $\left\langle\widehat{\aleph}_{7}^{n}, \mathbf{e}_{3 h}^{n+1 / 2}\right\rangle$ and $\left\langle\widehat{\aleph}_{8}^{n}, \mathbf{e}_{3 h}^{n+1 / 2}\right\rangle$, we proceed as follows. We estimate the first term as

$$
\begin{aligned}
\left|\left\langle\widehat{\aleph}_{7}^{n}, \mathbf{e}_{3 h}^{n+1 / 2}\right\rangle\right| & \leq 2 \sqrt{2} v_{r}\left\|\nabla \mathbf{e}_{1 h}^{n+1 / 2}\right\|\left\|\mathbf{e}_{3 h}\right\| \\
& \leq 4 v_{r}\left\|\mathbf{e}_{3 h}^{n+1 / 2}\right\|^{2}+\frac{\nu_{r}}{2}\left\|\nabla \mathbf{e}_{1 h}^{n+1 / 2}\right\|^{2}
\end{aligned}
$$

and the second as

$$
\begin{aligned}
\left|\left\langle\widehat{\aleph}_{8}^{n}, \mathbf{e}_{3 h}^{n+1 / 2}\right\rangle\right| \leq & c\left\|\mathbf{u}\left(t_{n+1 / 2}\right)-\overline{\mathbf{u}}\left(t_{n+1 / 2}\right)\right\|\left\|\nabla \mathbf{e}_{3 h}^{n+1 / 2}\right\| \\
\leq & c h^{2 k}\|(\mathbf{u}, p)\|_{\mathscr{C}\left(\left[t_{n}, t_{n+1}\right] ; \mathbf{H}^{k+1}(\Omega) \times \mathbf{H}^{k}(\Omega)\right)}^{2} \\
& +\frac{c_{a}+c_{d}}{8}\left\|\nabla \mathbf{e}_{3 h}^{n+1 / 2}\right\|^{2} .
\end{aligned}
$$

Applying estimates (44), (48), (49), (50), and (51) into (39) gives

$$
\begin{aligned}
& \left\|\mathbf{e}_{1 h}^{n+1}\right\|^{2}-\left\|\mathbf{e}_{1 h}^{n}\right\|^{2}+\frac{\left(\nu+v_{r}\right) \Delta t}{2}\left\|\nabla \mathbf{e}_{1 h}^{n+1 / 2}\right\|^{2} \leq \Delta t \Upsilon_{1}^{n} \\
& +c \Delta t\left\{\left\|\mathbf{e}_{1 h}^{n}\right\|^{2}+\left\|\mathbf{e}_{1 h}^{n-1}\right\|^{2}+\left\|\mathbf{e}_{3 h}^{n}\right\|^{2}+\left\|\mathbf{e}_{3 h}^{n-1}\right\|^{2}\right\} \\
& \left\|\mathbf{e}_{3 h}^{n+1}\right\|^{2}-\left\|\mathbf{e}_{3 h}^{n}\right\|^{2}+\frac{\left(c_{a}+c_{d}\right) \Delta t}{2}\left\|\nabla \mathbf{e}_{3 h}^{n+1 / 2}\right\|^{2} \\
& +\left(c_{0}+c_{d}-c_{a}\right) \Delta t\left\|\nabla \cdot \mathbf{e}_{3 h}^{n+1 / 2}\right\|^{2} \leq \Delta t \Upsilon_{2}^{n} \\
& \quad+c \Delta t\left[\left\|\mathbf{e}_{1 h}^{n}\right\|^{2}+\left\|\mathbf{e}_{1 h}^{n-1}\right\|^{2}\right]+\frac{v_{r}}{2} \Delta t\left\|\nabla \mathbf{e}_{1 h}^{n+1 / 2}\right\|^{2}
\end{aligned}
$$

where

$$
\begin{aligned}
Y_{1}^{n} & :=c\left\{(\Delta t)^{3}\left\|\partial_{t}^{3} \mathbf{u}\right\|_{L^{2}\left(t_{n}, t_{n+1} ; L^{2}(\Omega)\right)}^{2}\right. \\
& +\frac{h^{2 k}}{\Delta t}\left\|\left(\partial_{t} \mathbf{u}, \partial_{t} p\right)\right\|_{L^{2}\left(t_{n}, t_{n+1} ; \mathbf{H}^{k+1}(\Omega) \times \mathbf{H}^{k}(\Omega)\right)}^{2} \\
& +h^{2 k}\|(\mathbf{u}, p)\|_{\left.\mathscr{C}\left(t_{n}, t_{n+1}\right] ; \mathbf{H}^{k+1}(\Omega) \times \mathbf{H}^{k}(\Omega)\right)}^{2} \\
& +h^{2 k}\|\mathbf{w}\|_{\mathscr{C}\left(\left[t_{n}, t_{n+1}\right] ; \mathbf{H}^{k+1}(\Omega)\right)}^{2} \\
& \left.+(\Delta t)^{3}\left\|\left(\partial_{t}^{2} \mathbf{u}, \partial_{t}^{2} \mathbf{w}\right)\right\|_{L^{2}\left(t_{n}, t_{n+1} ; \mathbf{L}^{2}(\Omega)\right)}^{2}\right\} \\
Y_{2}^{n} & :=c\left\{(\Delta t)^{3}\left\|\partial_{t}^{3} \mathbf{w}\right\|_{L^{2}\left(t_{n}, t_{n+1} ; L^{2}(\Omega)\right)}^{2}\right. \\
& +\frac{h^{2 k}}{\Delta t}\left\|\partial_{t} \mathbf{w}\right\|_{L^{2}\left(t_{n}, t_{n+1} ; \mathbf{H}^{k+1}(\Omega)\right)}^{2} \\
& +h^{2 k}\|(\mathbf{u}, p)\|_{\left.\mathscr{C}\left(t_{n}, t_{n+1}\right] ; \mathbf{H}^{k+1}(\Omega) \times \mathbf{H}^{k}(\Omega)\right)}^{2} \\
& +h^{2 k}\|\mathbf{w}\|_{\mathscr{C}\left(\left[t_{n}, t_{n+1}\right] ; \mathbf{H}^{k+1}(\Omega)\right)}^{2} \\
& \left.+(\Delta t)^{3}\left\|\partial_{t}^{2} \mathbf{u}\right\|_{L^{2}\left(t_{n}, t_{n+1} ; \mathbf{L}^{2}(\Omega)\right)}^{2}\right\}
\end{aligned}
$$


Adding $(52)_{1}$ and $(52)_{2}$ yields

$$
\begin{aligned}
& \left\|\left(\mathbf{e}_{1 h}^{n+1}, \mathbf{e}_{3 h}^{n+1}\right)\right\|^{2}-\left\|\left(\mathbf{e}_{1 h}^{n}, \mathbf{e}_{3 h}^{n}\right)\right\|^{2}+\frac{\nu \Delta t}{2}\left\|\nabla \times \mathbf{e}_{1 h}^{n+1 / 2}\right\|^{2} \\
& \quad+\frac{\left(c_{a}+c_{d}\right)}{2} \Delta t\left\|\nabla \mathbf{e}_{3 h}^{n+1 / 2}\right\|^{2} \\
& +\left(c_{0}+c_{d}-c_{a}\right) \Delta t\left\|\nabla \cdot \mathbf{e}_{3 h}^{n+1 / 2}\right\|^{2} \\
& \leq c \Delta t\left[\left\|\left(\mathbf{e}_{1 h}^{n-1}, \mathbf{e}_{3 h}^{n-1}\right)\right\|^{2}+\left\|\left(\mathbf{e}_{1 h}^{n}, \mathbf{e}_{3 h}^{n}\right)\right\|^{2}\right]+\Delta t Y^{n},
\end{aligned}
$$

where

$$
\begin{aligned}
\Upsilon^{n} & :=\sum_{i=1}^{2} \Upsilon_{i}^{n}=c\left[(\Delta t)^{3}\left\|\left(\partial_{t}^{3} \mathbf{u}, \partial_{t}^{3} \mathbf{w}\right)\right\|_{L^{2}\left(t_{n}, t_{n+1} ; \mathbf{L}^{2}(\Omega)\right)}^{2}\right. \\
& +(\Delta t)^{3}\left\|\left(\partial_{t}^{2} \mathbf{u}, \partial_{t}^{2} \mathbf{w}\right)\right\|_{L^{2}\left(t_{n}, t_{n+1} ; L^{2}(\Omega)\right)}^{2}+\frac{h^{2 k}}{\Delta t} \|\left(\partial_{t} \mathbf{u}, \partial_{t} p\right. \\
& \left.\partial_{t} \mathbf{w}\right)\left\|_{L^{2}\left(t_{n}, t_{n+1} ; \mathbf{H}^{k+1}(\Omega) \times H^{k}(\Omega) \times \mathbf{H}^{k+1}(\Omega)\right)}^{2}+h^{2 k}\right\|(\mathbf{u}, \\
& p) \|_{\mathscr{C}\left(\left[t_{n}, t_{n+1}\right] ; \mathbf{H}^{k+1}(\Omega) \times H^{k}(\Omega)\right)}^{2} \\
& \left.+h^{2 k}\|\mathbf{w}\|_{\mathscr{C}\left(\left[t_{n}, t_{n+1}\right] ; \mathbf{H}^{k+1}(\Omega)\right)}^{2}\right]
\end{aligned}
$$

Summing (54) from $n=1$ to $m-1$ and applying the discrete Grönwall inequality, we have that

$$
\begin{gathered}
\left\|\mathbf{e}_{1 h}^{m}\right\|^{2}+\left\|\mathbf{e}_{3 h}^{m}\right\|^{2}+\frac{\Delta t \nu}{2} \sum_{n=1}^{m-1}\left\|\nabla \mathbf{e}_{1 h}^{n+1 / 2}\right\|^{2} \\
+\frac{\left(c_{a}+c_{d}\right) \Delta t}{2} \sum_{n=1}^{m-1}\left\|\nabla e_{3 h}^{n+1 / 2}\right\|^{2} \\
+\left(c_{0}+c_{d}-c_{a}\right) \Delta t \sum_{n=1}^{m-1}\left\|\nabla \cdot \mathbf{e}_{3 h}^{n+1 / 2}\right\|^{2} \\
\leq \Delta t \sum_{n=1}^{m-1} \Upsilon^{n}+\left\|\mathbf{e}_{1 h}^{0}\right\|^{2}+\left\|\mathbf{e}_{3 h}^{0}\right\|^{2} .
\end{gathered}
$$

Notice that from the assumptions on the solution $(\mathbf{u}, p, \mathbf{w})$ it holds that

$$
\Delta t \sum_{n=1}^{N} \Upsilon^{n} \leq c\left((\Delta t)^{4}+h^{2 k}\right) .
$$

Therefore the required error estimate now follows from (56), assumptions on the initial errors and triangle inequality.

We next analyze the convergence of pressure for the decoupled scheme. Note that

$$
\left\|p-p_{h}^{n}\right\| \leq\|p-\bar{p}\|+\left\|\bar{p}-p_{h}^{n}\right\|,
$$

so we need to only estimate $\left\|\bar{p}-p_{h}^{n}\right\|$.
Theorem 5. Under the assumptions in Theorem 4, the approximate pressure $p_{h}$ of (23) satisfies

$$
\left\|p-p_{h}\right\|_{l^{2}\left(L^{2}(\Omega)\right)} \leq \frac{c}{\sqrt{\Delta t}}\left(\Delta t^{2}+h^{k}\right),
$$

for some constant $c$ independent of mesh size $h$ and time step $\Delta t$.

Proof. From $(37)_{1}$ and inf-sup condition (17), it holds that

$$
\begin{aligned}
& \left\|e_{2 h}^{n+1 / 2}\right\| \leq \frac{1}{\beta} \sup _{\mathbf{v}_{h} \in \mathbf{X}_{h}} \frac{b\left(\mathbf{v}_{h}, e_{2 h}^{n+1 / 2}\right)}{\left\|\mathbf{v}_{h}\right\|_{1}} \leq \frac{1}{\beta} \sup _{\mathbf{v}_{h} \in \mathbf{X}_{h}} \frac{1}{\left\|\mathbf{v}_{h}\right\|_{1}} \\
& \cdot\left\{-\left(\mathscr{D} \mathbf{e}_{1 h}^{n+1}, \mathbf{v}_{h}\right)-a_{1}\left(\mathbf{e}_{1 h}^{n+1 / 2}, \mathbf{v}_{h}\right)\right. \\
& \left.+\left(\partial_{t} \mathbf{u}\left(t_{n+1 / 2}\right)-\mathscr{D} \overline{\mathbf{u}}\left(t_{n+1}\right), \mathbf{v}_{h}\right)+\left\langle\aleph_{h}^{n}, \mathbf{v}_{h}\right\rangle\right\} \\
& \quad \leq c\left\{\left\|\mathscr{D} \mathbf{e}_{1 h}^{n+1}\right\|+\left\|\nabla \mathbf{e}_{1 h}^{n+1 / 2}\right\|\right. \\
& \left.\quad+\left\|\partial_{t} \mathbf{u}\left(t_{n+1 / 2}\right)-\mathscr{D} \overline{\mathbf{u}}\left(t_{n+1}\right)\right\|_{X_{h}{ }^{*}}+\sum_{i=1}^{8}\left\|\aleph_{i}^{n}\right\|_{X_{h}{ }^{*}}\right\} .
\end{aligned}
$$

We start by estimating $\left\|\aleph_{5}^{n}\right\|_{X_{h}}$ and $\left\|\aleph_{6}^{n}\right\|_{X_{h}}$ as others can be estimated as usual. First, by Hölder's and Sobolev inequalities, we obtain

$$
\begin{aligned}
& \left|\left\langle\aleph_{5}^{n}, \mathbf{v}_{h}\right\rangle\right|=\left(\mathscr{I}\left(\overline{\mathbf{u}}\left(t_{n+1 / 2}\right)\right) \cdot \nabla \mathbf{e}_{1 h}^{n+1 / 2}, \mathbf{v}_{h}\right)+\frac{1}{2}(\nabla \\
& \left.\quad \cdot \mathscr{I}\left(\overline{\mathbf{u}}\left(t_{n+1 / 2}\right)\right) \mathbf{e}_{1 h}^{n+1 / 2} \mathbf{v}_{h}\right) \leq c\left(\left\|\mathscr{I}\left(\overline{\mathbf{u}}\left(t_{n+1 / 2}\right)\right)\right\|_{\infty}\right. \\
& \left.\quad+\left\|\nabla\left(\mathscr{I}\left(\overline{\mathbf{u}}\left(t_{n+1 / 2}\right)\right)\right)\right\|_{L^{3}(\Omega)}\right)\left\|\mathbf{e}_{1 h}^{n+1 / 2}\right\|_{1}\left\|\mathbf{v}_{h}\right\|_{1} .
\end{aligned}
$$

Before estimating the other term, notice that, by the inverse estimate (Assumption A2) and (56), we obtain

$$
\begin{aligned}
& \left\|\mathbf{e}_{1 h}^{n+1 / 2}\right\|_{1} \leq c^{*} \min \left\{h^{-1}\left\|\mathbf{e}_{1 h}^{n+1 / 2}\right\|,\left\|\mathbf{e}_{1 h}^{n+1 / 2}\right\|_{1}\right\} \\
& \quad \leq c \min \left\{h^{-1}\left(\Delta t^{2}+h^{k}\right),(\Delta t)^{-1}\left(\Delta t^{2}+h^{k}\right)\right\} \leq c .
\end{aligned}
$$

Therefore, by Hölder's and Sobolev inequalities and (62), we obtain

$$
\begin{aligned}
\left|\left\langle\aleph_{6}^{n}, \mathbf{v}_{h}\right\rangle\right| & \leq c\left\|\mathscr{I}\left(\mathbf{e}_{1 h}^{n+1 / 2}\right)\right\|_{1}\left\|\mathbf{e}_{1 h}^{n+1 / 2}\right\|_{1}\left\|\mathbf{v}_{h}\right\|_{1} \\
& \leq c^{*}\left\|\mathscr{I}\left(\mathbf{e}_{1 h}^{n+1 / 2}\right)\right\|_{1}\left\|\mathbf{v}_{h}\right\|_{1} .
\end{aligned}
$$

Estimating other terms in (60) as we did in the proof of Theorem 4, we obtain

$$
\begin{aligned}
& \left\|e_{2 h}^{n+1 / 2}\right\| \leq c\left\{\left\|\mathscr{D} \mathbf{e}_{1 h}^{n+1}\right\|+\left\|\nabla \mathbf{e}_{1 h}^{n+1 / 2}\right\|+\left\|\nabla \mathbf{e}_{3 h}^{n+1 / 2}\right\|\right. \\
& +\left\|\mathscr{I}\left(\mathbf{e}_{1 h}^{n+1 / 2}\right)\right\|_{1}+\left\|\mathscr{I}\left(\mathbf{e}_{3 h}^{n+1 / 2}\right)\right\|+(\Delta t)^{3 / 2}+h^{k} \\
& \left.+\frac{h^{k}}{\sqrt{\Delta t}}\right\} .
\end{aligned}
$$

The required error estimate now follows from last inequality by using Theorem 4 and triangle inequality. 
The error estimate for the pressure in the previous theorem can be improved under stronger regularity properties of the solution. To this end, we next derive optimal order error estimates for the time derivatives of velocity and micropolar velocity.

Corollary 6. Suppose the assumptions of Theorem 4 hold. Moreover, assume $\mathbf{u}, \mathbf{w} \in H^{2}\left(0, T ; \mathbf{H}^{1}(\Omega)\right)$ and the initial conditions satisfy $\sum_{i=0}^{1}\left\|\left(\mathbf{u}\left(t_{i}\right), \mathbf{w}\left(t_{i}\right)\right)-\left(\mathbf{u}_{h}^{i}, \mathbf{w}_{h}^{i}\right)\right\|_{1} \leq c h^{k}$ and $b\left(\mathbf{u}_{h}^{i}, r_{h}\right)=0, \forall r_{h} \in Q_{h}$, for $i=0,1$. Then for any $h \in\left(0, h_{0}\right]$ the approximations $\mathbf{u}_{h}^{n}$ and $\mathbf{w}_{h}^{n}$ satisfy

$$
\begin{aligned}
& \left\|\partial_{t} \mathbf{u}-\mathscr{D} \mathbf{u}_{h}\right\|_{l^{2}\left(L^{2}(\Omega)\right)},\left\|\partial_{t} \mathbf{w}-\mathscr{D} \mathbf{w}_{h}\right\|_{l^{2}\left(L^{2}(\Omega)\right)} \\
& \quad \leq c\left(\Delta t^{2}+h^{k}\right) .
\end{aligned}
$$

Moreover, we have

$$
\left\|\mathbf{u}-\mathbf{u}_{h}\right\|_{l^{\infty}\left(H^{1}(\Omega)\right)},\left\|\mathbf{w}-\mathbf{w}_{h}\right\|_{l^{\infty}\left(H^{1}(\Omega)\right)} \leq c\left(\Delta t^{2}+h^{k}\right),
$$

for some constant $c$ independent of the mesh size $h$ and time step $\Delta t$.

Proof. Putting $\left(\mathbf{v}_{h}, \phi_{h}\right)=\left(\mathscr{D}\left(\mathbf{e}_{1 h}^{n+1}\right), \mathscr{D}\left(\mathbf{e}_{3 h}^{n+1}\right)\right)$ into (37) and splitting the nonlinear terms as in the proof of Theorem 4, we obtain

$$
\begin{aligned}
& \| \mathscr{D}\left(\mathbf{e}_{1 h}^{n+1}\right) \|^{2}+\frac{\left(\nu+v_{r}\right)}{2} \mathscr{D}\left(\left\|\nabla \mathbf{e}_{1 h}^{n+1}\right\|^{2}\right) \\
&=\left(\partial_{t} \mathbf{u}\left(t_{n+1 / 2}\right)-\mathscr{D}\left(\overline{\mathbf{u}}\left(t_{n+1}\right)\right), \mathscr{D}\left(\mathbf{e}_{1 h}^{n+1}\right)\right) \\
&+\sum_{i=1}^{8}\left\langle\aleph_{i}^{n}, \mathscr{D}\left(\mathbf{e}_{1 h}^{n+1}\right)\right\rangle, \\
&\left\|\mathscr{D}\left(\mathbf{e}_{3 h}^{n}\right)\right\|^{2}+\frac{\left(c_{a}+c_{d}\right)}{2} \mathscr{D}\left(\left\|\nabla \mathbf{e}_{3 h}^{n+1}\right\|^{2}\right) \\
& \quad+\frac{\left(c_{0}+c_{d}\right)}{2} \mathscr{D}\left(\left\|\nabla \cdot \mathbf{e}_{3 h}^{n+1}\right\|^{2}\right)+2 v_{r} \mathscr{D}\left(\left\|\mathbf{e}_{3 h}^{n+1}\right\|^{2}\right) \\
& \quad=\left(\partial_{t} \mathbf{w}\left(t_{n+1 / 2}\right)-\mathscr{D}\left(\overline{\mathbf{w}}\left(t_{n+1}\right)\right), \mathscr{D}\left(\mathbf{e}_{3 h}^{n+1}\right)\right) \\
& \quad+\sum_{i=1}^{8}\left\langle\widehat{\aleph}_{i}^{n}, \mathscr{D}\left(\mathbf{e}_{3 h}^{n+1}\right)\right\rangle \\
& \quad+4 v_{r}\left(\mathbf{w}\left(t_{n+1 / 2}\right)-\overline{\mathbf{w}}\left(t_{n+1 / 2}\right), \mathscr{D}\left(\mathbf{e}_{3 h}^{n+1}\right)\right) .
\end{aligned}
$$

Next, we bound each of the terms on the right-hand sides of (67). We start by estimating $\left\langle\aleph_{i}^{n}, \mathscr{D}\left(\mathbf{e}_{1 h}^{n+1}\right)\right\rangle$ for $i=1, \ldots, 8$ in $(67)_{1}$. To this end, we use Hölder's inequality and Sobolev inequality. We find that

$$
\begin{aligned}
& \left|\left\langle\aleph_{1}^{n}, \mathscr{D}\left(\mathbf{e}_{1 h}^{n+1}\right)\right\rangle\right| \\
& \quad \leq c\left(\left\|\mathbf{u}\left(t_{n+1 / 2}\right)\right\|_{\infty}+\left\|\nabla \mathbf{u}\left(t_{n+1 / 2}\right)\right\|_{L^{3}(\Omega)}\right) \\
& \cdot\left\|\mathbf{u}\left(t_{n+1 / 2}\right)-\overline{\mathbf{u}}\left(t_{n+1 / 2}\right)\right\|_{1}\left\|\mathscr{D}\left(\mathbf{e}_{1 h}^{n+1}\right)\right\|,
\end{aligned}
$$

$$
\begin{aligned}
& \left|\left\langle\aleph_{2}^{n}, \mathscr{D}\left(\mathbf{e}_{1 h}^{n+1}\right)\right\rangle\right| \leq c\left\|\mathbf{u}\left(t_{n+1 / 2}\right)-\mathscr{I}\left(\mathbf{u}\left(t_{n+1 / 2}\right)\right)\right\|_{1} \\
& \cdot\left(\left\|\overline{\mathbf{u}}\left(t_{n+1 / 2}\right)\right\|_{\infty}+\left\|\nabla \overline{\mathbf{u}}\left(t_{n+1 / 2}\right)\right\|_{L^{3}(\Omega)}\right)\left\|\mathscr{D}\left(\mathbf{e}_{1 h}^{n+1}\right)\right\|, \\
& \left|\left\langle\aleph_{3}^{n}, \mathscr{D}\left(\mathbf{e}_{1 h}^{n+1}\right)\right\rangle\right| \\
& \leq c\left(\left\|\overline{\mathbf{u}}\left(t_{n+1 / 2}\right)\right\|_{\infty}+\left\|\nabla \overline{\mathbf{u}}\left(t_{n+1 / 2}\right)\right\|_{L^{3}(\Omega)}\right) \\
& \cdot\left\|\mathscr{I}\left(\mathbf{u}\left(t_{n+1 / 2}\right)-\overline{\mathbf{u}}\left(t_{n+1 / 2}\right)\right)\right\|_{1}\left\|\mathscr{D}\left(\mathbf{e}_{1 h}^{n+1}\right)\right\|, \\
& \left|\left\langle\aleph_{4}^{n}, \mathscr{D}\left(\mathbf{e}_{1 h}^{n+1}\right)\right\rangle\right| \\
& \leq c\left(\left\|\overline{\mathbf{u}}\left(t_{n+1 / 2}\right)\right\|_{\infty}+\left\|\nabla \overline{\mathbf{u}}\left(t_{n+1 / 2}\right)\right\|_{L^{3}(\Omega)}\right) \\
& \cdot\left\|\mathscr{I}\left(\mathbf{e}_{1 h}^{n+1 / 2}\right)\right\|_{1}\left\|\mathscr{D}\left(\mathbf{e}_{1 h}^{n+1}\right)\right\|, \\
& \left|\left\langle\aleph_{5}^{n}, \mathscr{D}\left(\mathbf{e}_{1 h}^{n+1}\right)\right\rangle\right| \\
& \leq c\left(\left\|\mathscr{I}\left(\overline{\mathbf{u}}\left(t_{n+1 / 2}\right)\right)\right\|_{\infty}+\left\|\nabla \mathscr{I}\left(\overline{\mathbf{u}}\left(t_{n+1 / 2}\right)\right)\right\|_{L^{3}(\Omega)}\right) \\
& \cdot\left\|\mathbf{e}_{1 h}^{n+1 / 2}\right\|_{1}\left\|\mathscr{D}\left(\mathbf{e}_{1 h}^{n+1}\right)\right\| .
\end{aligned}
$$

From the inverse inequality (Assumption A2) and Sobolev inequality, it follows that

$$
\left\|\phi_{h}\right\|_{\infty}+\left\|\nabla \phi_{h}\right\|_{L^{3}(\Omega)} \leq c h^{-d / 6}\left\|\phi_{h}\right\|_{1} \quad \forall \phi_{h} \in X^{h} .
$$

Using (69), we estimate $\left\langle\aleph_{6}^{n}, \mathscr{D}\left(\mathbf{e}_{1 h}^{n+1}\right)\right\rangle$ as below

$$
\begin{aligned}
& \left|\left\langle\aleph_{6}^{n}, \mathscr{D}\left(\mathbf{e}_{1 h}^{n+1}\right)\right\rangle\right| \\
& \leq\left[\left\|\mathscr{I}\left(\mathbf{e}_{1 h}^{n+1 / 2}\right)\right\|_{\infty}+\left\|\nabla \mathscr{I}\left(\mathbf{e}_{1 h}^{n+1 / 2}\right)\right\|_{L^{3}(\Omega)}\right]\left\|\mathbf{e}_{1 h}^{n+1 / 2}\right\|_{1} \\
& \cdot\left\|\mathscr{D}\left(\mathbf{e}_{1 h}^{n+1}\right)\right\| \leq c^{*}\left\|\mathbf{e}_{1 h}^{n+1 / 2}\right\|_{1}\left\|\mathscr{I}\left(\mathbf{e}_{1 h}^{n+1 / 2}\right)\right\|_{1} \\
& \cdot h^{-d / 6}\left\|\mathscr{D}\left(\mathbf{e}_{1 h}^{n+1}\right)\right\| .
\end{aligned}
$$

Alternatively, we can estimate $\left\langle\aleph_{6}^{n}, \mathscr{D}\left(\mathbf{e}_{1 h}^{n}\right)\right\rangle$ as follows:

$$
\begin{aligned}
\left|\left\langle\aleph_{6}^{n}, \mathscr{D}\left(\mathbf{e}_{1 h}^{n+1}\right)\right\rangle\right|= & \left|\frac{1}{2 \Delta t} c_{1}\left(\mathscr{I}\left(\mathbf{e}_{1 h}^{n+1 / 2}\right), \mathbf{e}_{1 h}^{n+1}, \mathbf{e}_{1 h}^{n}\right)\right| \\
& +\left|\frac{1}{2 \Delta t} c_{1}\left(\mathscr{I}\left(\mathbf{e}_{1 h}^{n+1 / 2}\right), \mathbf{e}_{1 h}^{n}, \mathbf{e}_{1 h}^{n+1}\right)\right| \\
\leq & \frac{c^{*}}{\Delta t}\left\|\mathscr{I}\left(\mathbf{e}_{1 h}^{n+1 / 2}\right)\right\|_{1}\left\|\mathbf{e}_{1 h}^{n}\right\|_{1}\left\|\mathbf{e}_{1 h}^{n+1}\right\|_{1} .
\end{aligned}
$$

Combining (70) and (71), we have

$$
\begin{aligned}
& \quad\left|\left\langle\aleph_{6}^{n}, \mathscr{D}\left(\mathbf{e}_{1 h}^{n+1}\right)\right\rangle\right| \\
& \quad \leq c \gamma_{n}\left\|\mathscr{I}\left(\mathbf{e}_{1 h}^{n+1 / 2}\right)\right\|_{1}\left[\left\|\mathscr{D}\left(\mathbf{e}_{1 h}^{n+1}\right)\right\|+\left\|\mathbf{e}_{1 h}^{n-1}\right\|_{1}\right],
\end{aligned}
$$

where

$$
\gamma_{n}:=\min \left\{h^{-d / 6},(\Delta t)^{-1 / 2}\right\}\left\|\mathbf{e}_{1 h}^{n+1 / 2}\right\|_{1} .
$$


Estimating the other terms similarly, we obtain

$$
\begin{gathered}
\left|\left\langle\aleph_{7}^{n}, \mathscr{D}\left(\mathbf{e}_{1 h}^{n+1}\right)\right\rangle\right| \leq c\left\|\mathscr{I}\left(\overline{\mathbf{w}}\left(t_{n+1 / 2}\right)\right)-\mathbf{w}\left(t_{n+1 / 2}\right)\right\|_{1} \\
\cdot\left\|\mathscr{D}\left(\mathbf{e}_{1 h}^{n+1}\right)\right\| \leq c\left[h^{k}\|\mathbf{w}\|_{C\left(\left[t_{n-1}, t_{n+1}\right] ; \mathbf{H}^{k+1}(\Omega)\right)}\right. \\
\left.+(\Delta t)^{3 / 2}\left\|\partial_{t}^{2} \mathbf{w}\right\|_{L^{2}\left(t_{n}, t_{n+1 / 2} ; \mathbf{H}^{1}(\Omega)\right)}\right]\left\|\mathscr{D}\left(\mathbf{e}_{1 h}^{n+1}\right)\right\|, \\
\left|\left\langle\aleph_{8}^{n}, \mathscr{D}\left(\mathbf{e}_{1 h}^{n+1}\right)\right\rangle\right| \leq c\left\|\nabla \mathscr{I}\left(\mathbf{e}_{3 h}^{n+1 / 2}\right)\right\|\left\|\mathscr{D}\left(\mathbf{e}_{1 h}^{n+1}\right)\right\| .
\end{gathered}
$$

We procced similarly for the terms in $(67)_{2}$. Estimation of $\left\langle\widehat{\aleph}_{1}^{n}, \mathscr{D}\left(\mathbf{e}_{3 h}^{n+1}\right)\right\rangle-\left\langle\widehat{\aleph}_{5}^{n}, \mathscr{D}\left(\mathbf{e}_{3 h}^{n+1}\right)\right\rangle$ is similar to estimation of $\left\langle\aleph_{1}^{n}, \mathscr{D}\left(\mathbf{e}_{1 h}^{n+1}\right)\right\rangle-\left\langle\aleph_{5}^{n}, \mathscr{D}\left(\mathbf{e}_{1 h}^{n+1}\right)\right\rangle$. We obtain

$$
\begin{aligned}
\sum_{i=1}^{5} & \left|\left\langle\widehat{\aleph}_{i}^{n}, \mathscr{D}\left(\mathbf{e}_{3 h}^{n+1}\right)\right\rangle\right| \leq c\left[\left\|\mathbf{w}\left(t_{n+1 / 2}\right)-\overline{\mathbf{w}}\left(t_{n+1 / 2}\right)\right\|_{1}\right. \\
& +\left\|\mathbf{w}\left(t_{n+1 / 2}\right)-\mathscr{I}\left(\mathbf{w}\left(t_{n+1 / 2}\right)\right)\right\|_{1} \\
& +\left\|\mathscr{I}\left(\mathbf{u}\left(t_{n+1 / 2}\right)-\overline{\mathbf{w}}\left(t_{n+1 / 2}\right)\right)\right\|\left\|\mathbf{e}_{3 h}^{n+1 / 2}\right\|_{1} \\
& \left.+\left\|\mathscr{I}\left(\mathbf{e}_{1 h}^{n+1 / 2}\right)\right\|_{1}\right]\left\|\mathscr{D}\left(\mathbf{e}_{1 h}^{n+1}\right)\right\| .
\end{aligned}
$$

We estimate $\left\langle\widehat{\aleph}_{6}^{n}, \mathscr{D}\left(\mathbf{e}_{3 h}^{n+1}\right)\right\rangle$ in the same way we estimated $\left\langle\aleph_{6}^{n}, \mathscr{D}\left(\mathbf{e}_{1 h}^{n+1}\right)\right\rangle$. We obtain

$$
\begin{aligned}
& \left|\left\langle\widehat{\aleph}_{6}^{n}, \mathscr{D}\left(\mathbf{e}_{3 h}^{n+1}\right)\right\rangle\right| \\
& \quad \leq c \widehat{\gamma}_{n}\left\|\mathscr{I}\left(\mathbf{e}_{1 h}^{n+1 / 2}\right)\right\|_{1}\left[\left\|\mathscr{D}\left(\mathbf{e}_{3 h}^{n+1}\right)+\right\| \mathbf{e}_{3 h}^{n-1} \|_{1}\right],
\end{aligned}
$$

where

$$
\widehat{\gamma}_{n}:=\min \left\{h^{-d / 6},(\Delta t)^{-1 / 2}\right\}\left\|e_{3 h}^{n+1 / 2}\right\|_{1} .
$$

Estimating other terms as usual, we obtain

$$
\begin{aligned}
& \left|\sum_{i=7}^{8}\left\langle\widehat{\aleph}_{i}^{n}, \mathscr{D}\left(\mathbf{e}_{3 h}^{n+1}\right)\right\rangle\right| \\
& \quad \leq c\left[\left\|\nabla \mathbf{e}_{1 h}^{n+1 / 2}\right\|+\left\|\overline{\mathbf{u}}\left(t_{n+1 / 2}\right)-\mathbf{u}\left(t_{n+1 / 2}\right)\right\|_{1}\right] \\
& \quad \cdot\left\|\mathscr{D}\left(\mathbf{e}_{3 h}^{n+1}\right)\right\| .
\end{aligned}
$$

Employing these estimates in (67), we can write it as

$$
\begin{aligned}
& \left(\left\|\nabla \mathbf{e}_{1 h}^{n+1}\right\|^{2}-\left\|\nabla \mathbf{e}_{1 h}^{n}\right\|^{2}\right)+\frac{\Delta t}{\left(\nu_{r}+v\right)}\left\|\mathscr{D}\left(\mathbf{e}_{1 h}^{n+1}\right)\right\|^{2} \\
& \leq c\left[\gamma_{n}^{2} \Delta t\left\|\mathscr{I}\left(\mathbf{e}_{1 h}^{n+1 / 2}\right)\right\|_{1}^{2}+\alpha_{n} \Delta t\right] \\
& \left(\left\|\nabla \mathbf{e}_{3 h}^{n+1}\right\|^{2}-\left\|\nabla \mathbf{e}_{3 h}^{n}\right\|^{2}\right) \\
& +\frac{\left(c_{0}+c_{d}\right)}{\left(c_{a}+c_{d}\right)}\left(\left\|\nabla \cdot \mathbf{e}_{3 h}^{n+1}\right\|^{2}-\left\|\nabla \cdot \mathbf{e}_{3 h}^{n}\right\|^{2}\right) \\
& +\frac{4 v_{r}}{\left(c_{a}+c_{d}\right)}\left(\left\|\mathbf{e}_{3 h}^{n+1}\right\|^{2}-\left\|\mathbf{e}_{3 h}^{n}\right\|^{2}\right)
\end{aligned}
$$

$$
\begin{aligned}
& +\frac{\Delta t}{\left(c_{a}+c_{d}\right)}\left\|\mathscr{D}\left(\mathbf{e}_{3 h}^{n+1}\right)\right\|^{2} \\
& \leq c\left[\widehat{\alpha}_{n} \Delta t+\gamma_{n}^{2} \Delta t\left\|\mathscr{I}\left(\mathbf{e}_{1 h}^{n}\right)\right\|_{1}^{2}\right],
\end{aligned}
$$

where

$$
\begin{aligned}
& \alpha_{n}:=(\Delta t)^{3}\left\|\partial_{t}^{3} \mathbf{u}\right\|_{L^{2}\left(t_{n}, t_{n+1} ; \mathbf{L}^{2}(\Omega)\right)}^{2} \\
& +\frac{h^{2 k}}{\Delta t}\left\|\left(\partial_{t} \mathbf{u}, \partial_{t} p\right)\right\|_{L^{2}\left(t_{n}, t_{n+1} ; \mathbf{H}^{k+1}(\Omega) \times H^{k}(\Omega)\right)}^{2} \\
& +h^{2 k}\|(\mathbf{u}, p)\|_{\mathscr{C}\left(\left[t_{n}, t_{n+1}\right] ; \mathbf{H}^{k+1}(\Omega) \times H^{k}(\Omega)\right)}^{2} \\
& +h^{2 k}\|\mathbf{w}\|_{\mathscr{C}\left(\left[t_{n}, t_{n+1}\right] ; \mathbf{H}^{k+1}(\Omega)\right)}^{2} \\
& +(\Delta t)^{3}\left\|\partial_{t}^{2} \mathbf{u}\right\|_{L^{2}\left(t_{n}, t_{n+1} ; \mathbf{H}^{1}(\Omega)\right)}^{2} \\
& +(\Delta t)^{3}\left\|\partial_{t}^{2} \mathbf{w}\right\|_{L^{2}\left(t_{n}, t_{n+1} ; \mathbf{H}^{1}(\Omega)\right)}^{2} \\
& +\sum_{i=0}^{1}\left[\left\|\mathbf{e}_{1 h}^{n-i}\right\|_{1}^{2}+\left\|\mathbf{e}_{4 h}^{n-i}\right\|_{1}^{2}\right]+\left\|\mathbf{e}_{3 h}^{n+1 / 2}\right\|_{1}^{2} \\
& +\left\|\mathbf{e}_{1 h}^{n+1 / 2}\right\|_{1}^{2}, \\
& \widehat{\alpha}_{n}:=(\Delta t)^{3}\left\|\partial_{t}^{3} \mathbf{w}\right\|_{L^{2}\left(t_{n}, t_{n+1} ; L^{2}(\Omega)\right)}^{2} \\
& +\frac{h^{2 k}}{\Delta t}\left\|\partial_{t} \mathbf{w}\right\|_{L^{2}\left(t_{n}, t_{n+1} ; \mathbf{H}^{k+1}(\Omega)\right)}^{2} \\
& +h^{2 k}\|(\mathbf{u}, p)\|_{\mathscr{C}\left(\left[t_{n}, t_{n+1}\right] ; \mathbf{H}^{k+1}(\Omega) \times H^{k}(\Omega)\right)}^{2} \\
& +h^{2 k}\|\mathbf{w}\|_{\mathscr{C}\left(\left[t_{n}, t_{n+1}\right] ; \mathbf{H}^{k+1}(\Omega)\right)}^{2} \\
& +(\Delta t)^{3}\left\|\partial_{t}^{2} \mathbf{w}\right\|_{L^{2}\left(t_{n}, t_{n+1} ; \mathbf{H}^{1}(\Omega)\right)}^{2}+\sum_{i=0}^{2}\left\|\mathbf{e}_{1 h}^{n-i}\right\|_{1}^{2} \\
& +\left\|\mathbf{e}_{3 h}^{n+1 / 2}\right\|_{1}^{2}+\left\|\mathbf{e}_{1 h}^{n+1 / 2}\right\|_{1}^{2} .
\end{aligned}
$$

Notice, by the regularity properties of the solution $(\mathbf{u}, p, \mathbf{w})$ and Theorem 4, we have

$$
\Delta t \sum_{i=1}^{N} \alpha_{i}, \Delta t \sum_{i=1}^{N} \widehat{\alpha}_{i} \leq c\left((\Delta t)^{4}+h^{2 k}\right) .
$$

Therefore summing (79) from $n=1$ to $m-1$ and using (81) and the assumptions on initial conditions $\left(\mathbf{u}_{h}^{i}, \mathbf{w}_{h}^{i}\right), i=0,1$, we obtain

$$
\begin{aligned}
& \left\|\nabla \mathbf{e}_{1 h}^{m}\right\|^{2}+\frac{\Delta t}{\left(v_{r}+v\right)} \sum_{n=1}^{m-1}\left\|\mathscr{D}\left(\mathbf{e}_{1 h}^{n+1}\right)\right\|^{2} \\
& \leq c\left[\sum_{n=1}^{m-1} \gamma_{n}^{2} \Delta t\left\|\nabla \mathbf{e}_{1 h}^{n}\right\|^{2}+(\Delta t)^{4}+h^{2 k}\right], \\
& \left\|\nabla \mathbf{e}_{3 h}^{m}\right\|^{2}+\frac{\left(c_{0}+c_{d}\right)}{\left(c_{a}+c_{d}\right)}\left\|\nabla \cdot \mathbf{e}_{3 h}^{m}\right\|^{2}+\frac{4 v_{r}}{\left(c_{a}+c_{d}\right)}\left\|\mathbf{e}_{3 h}^{m}\right\|^{2}
\end{aligned}
$$




$$
\begin{aligned}
& +\frac{\Delta t}{\left(c_{a}+c_{d}\right)} \sum_{n=1}^{m-1}\left\|\mathscr{D}\left(\mathbf{e}_{3 h}^{n+1}\right)\right\|^{2} \\
& \leq c\left[(\Delta t)^{4}+h^{2 k}+\sum_{n=1}^{m-1} \hat{\gamma}_{n}^{2} \Delta t\left\|\mathscr{I}\left(\mathbf{e}_{1 h}^{n}\right)\right\|_{1}^{2}\right] .
\end{aligned}
$$

Notice that, by Theorem 4 and the definition of $\gamma_{n}$ in (73), we have that

$$
\begin{aligned}
\Delta t \sum_{i=1}^{N} \gamma_{i}^{2} & \leq \min \left\{h^{-d / 3},(\Delta t)^{-2}\right\} \Delta t \sum_{i=1}^{N}\left\|\mathrm{e}_{1 h}^{i}\right\|_{1}^{2} \\
& \leq c \min \left\{h^{-d / 3},(\Delta t)^{-2}\right\}\left(h^{2 k}+(\Delta t)^{4}\right) \\
& \leq c \min \left\{h^{2 k-d / 3}+(\Delta t)^{2}\right\} \leq c .
\end{aligned}
$$

Similarly, using the definition of $\widehat{\gamma}_{n}$ in (77), we can show that

$$
\Delta t \sum_{i=1}^{N} \widehat{\gamma}_{i}^{2} \leq c
$$

The required results now follow if we add $(82)_{1}$ and $(82)_{2}$ and apply the discrete Grönwall inequality to the resulting inequality with (83)-(84).

Corollary 7. Suppose the assumptions of Corollary 6 hold. Then the approximate pressure $p_{h}^{n+1 / 2}$ in (23) satisfies

$$
\left\|p-p_{h}\right\|_{l^{2}\left(L^{2}(\Omega)\right)} \leq c\left(\Delta t^{2}+h^{k}\right)
$$

Proof. We provide only a sketch of the proof of this corollary as it is similar to the proof of Theorem 5. It follows from (82) that

$$
\Delta t\left\|\mathscr{D} \mathbf{e}_{1 h}^{n+1}\right\|^{2} \leq c\left((\Delta t)^{4}+h^{2 k}\right)
$$

Therefore using (86) in (64), we obtain the required estimate.

Remark 8. The error estimates we have obtained so far also provide stability estimates without any time step restriction. In particular, it proves that the fully discrete velocities and microrotation vector fields are bounded in $\ell^{\infty}\left(\mathbf{H}^{1}(\Omega)\right)$ and the pressures are bounded in $\ell^{2}\left(\mathbf{L}^{2}(\Omega)\right)$ due to the regularity assumed on the continuous solutions.

\section{Numerical Results}

In this section, we present numerical results from tests which confirm the theoretical convergence rates of our algorithm. Assume the spatial domain $\Omega=[0,1] \times[0,1]$ and the time
TABLE 1: Convergence performance of the decoupled and monolithic (coupled) schemes at time $t_{N}=1.0$, with fixed time step $\Delta t=0.01$.

\begin{tabular}{lcccc}
\hline & \multicolumn{2}{c}{ Coupled scheme } & \multicolumn{2}{c}{ Decoupled scheme } \\
$h$ & $\left\|\mathbf{u}\left(t_{n}\right)-\mathbf{u}_{h}^{n}\right\|$ & $\left\|\mathbf{w}\left(t_{n}\right)-\mathbf{w}_{h}^{n}\right\|$ & $\left\|\mathbf{u}\left(t_{n}\right)-\mathbf{u}_{h}^{n}\right\|$ & $\left\|\mathbf{w}\left(t_{n}\right)-\mathbf{w}_{h}^{n}\right\|$ \\
\hline $1 / 2$ & 0.03508120 & 0.0224123 & 0.0350728 & 0.0219623 \\
$1 / 4$ & 0.0086203 & 0.00580328 & 0.0087782 & 0.0055905 \\
$1 / 8$ & 0.0024316 & 0.00150977 & 0.0022925 & 0.0013795 \\
$1 / 16$ & 0.00058251 & 0.000036079 & 0.0005587 & 0.00034529 \\
$1 / 32$ & 0.00012395 & 0.000088654 & 0.00014729 & 0.00008624 \\
\hline
\end{tabular}

interval $0 \leq t \leq 1$. We use the exact solution $(\mathbf{u}, p, \mathbf{w})$ given by

$$
\begin{aligned}
\mathbf{u}= & \left(-(1-\cos (2 \pi x)) \sin (2 \pi y) e^{-t}, \sin (2 \pi y)\right. \\
& \left.\cdot(1-\cos (2 \pi y)) e^{-t}\right), \\
\mathbf{w}= & \left(\cos (\pi y) \sin (\pi x) e^{-t},-\sin (\pi y) \cos (\pi x) e^{-t}\right), \\
p= & (\sin (4 \pi x)+\sin (4 \pi y)) e^{-t} .
\end{aligned}
$$

Here the source terms, initial conditions, and boundary conditions are chosen to correspond to the exact solution. The finite element spaces are constructed using piecewise quadratic polynomial for velocity and piecewise linear polynomial for the pressure in the Navier-Stokes equations and quadratic finite elements for the microrotational velocity. The performance of the numerical scheme studied herein is also compared with the monolithic, fully implicit method derived by setting $\mathscr{I}\left(\mathbf{u}_{h}^{n+1 / 2}\right)=\mathbf{u}_{h}^{n+1 / 2}$ and $\mathscr{I}\left(\mathbf{w}_{h}^{n+1 / 2}\right)=\mathbf{w}_{h}^{n+1 / 2}$ in Algorithm 3. The monolithic scheme requires a system of nonlinear algebraic equations to be solved using an iterative method at each time step. We employ Newton iterative method for solving those nonlinear algebraic equations and the iteration is stopped when relative nonlinear residual is less than $10^{-6}$.

In Table 1, we consider both schemes at time $t_{N}=1.0$, with varying spacing $h$ but for fixed time step $\Delta t=0.01$.

The results in Table 1 show that the two schemes achieve similar precision. Moreover, it can be seen that the error estimates of $\mathbf{u}$ and $\mathbf{w}$ in Theorem 4 for the order of convergence in space agree well with the numerical experiments. In order to determine the order of convergence $\alpha$ with respect to the time step $\Delta t$, we will use the following approximation:

$$
\alpha \approx \log _{2} \frac{\left\|\mathbf{v}_{h, \Delta t}\left(x, t_{N}\right)-\mathbf{v}_{h, \Delta t / 2}\left(x, t_{N}\right)\right\|}{\left\|\mathbf{v}_{h, \Delta t / 2}\left(x, t_{N}\right)-\mathbf{v}_{h, \Delta t / 4}\left(x, t_{N}\right)\right\|}
$$

In Table 2, we list the values of the right-hand side of (88) with fixed spacing $h=1 / 32$ and varying time step $\Delta t=1 / 20,1 / 40,1 / 80,1 / 160$. As can be seen the orders of convergence in time are all of second order for the decoupled scheme suggesting that the orders of convergence in time in error estimates in Theorem 4 for the $L^{2}$-norm of $\mathbf{u}$ and $\mathbf{w}$ are optimal. 
TABLE 2: Convergence orders of $O\left(\Delta t^{\alpha}\right)$ of the decoupled scheme at time $t_{N}=1.0$, with the fixed spacing $h=1 / 32$.

\begin{tabular}{lcccc}
\hline$\Delta t$ & $\left\|\mathbf{u}\left(t_{n}\right)-\mathbf{u}_{h}^{n}\right\|$ & Rate & $\left\|\mathbf{w}\left(t_{n}\right)-\mathbf{w}_{h}^{n}\right\|$ & Rate \\
\hline $1 / 20$ & $4.01235 \times 10^{-5}$ & - & $3.96243 \times 10^{-5}$ & - \\
$1 / 40$ & $2.0319915 \times 10^{-5}$ & 1.97459 & $2.001783 \times 10^{-5}$ & 1.97945 \\
$1 / 80$ & $1.0221336 \times 10^{-5}$ & 1.98799 & $1.0067712 \times 10^{-5}$ & 1.98832 \\
$1 / 160$ & $5.126534 \times 10^{-6}$ & 1.99381 & $5.047737 \times 10^{-6}$ & 1.9945 \\
\hline
\end{tabular}

4.1. Conclusion. In this paper, we give a complete error analysis of an efficient time-stepping scheme for micropolar fluid flow problems. Our algorithm extrapolates the coupling terms to the previous time levels at each time step and solves each subphysics problem separately without iteration. We derived optimal order error estimates in suitable norms without assuming any time step restriction. These error estimates also show the scheme is unconditionally stable. Numerical tests illustrate the validity of the theoretical results.

\section{Competing Interests}

The author declares that there are no competing interests.

\section{References}

[1] D. B. Holmes and J. R. Vermeulen, "Velocity profiles in ducts with rectangular cross sections," Chemical Engineering Science, vol. 23, no. 7, pp. 717-722, 1968.

[2] I. Papautsky, J. Brazzle, T. Ameel, and A. B. Frazier, "Laminar fluid behavior in microchannels using micropolar fluid theory," Sensors and Actuators A: Physical, vol. 73, no. 1-2, pp. 101-108, 1999.

[3] W. Peiyi and W. A. Little, "Measurement of friction factors for the flow of gases in very fine channels used for microminiature Joule-Thomson refrigerators," Cryogenics, vol. 23, no. 5, pp. 273277, 1983.

[4] P. Wilding, J. Pfahler, H. H. Bau, J. N. Zemel, and L. J. Kricka, "Manipulation and flow of biological fluids in straight channels micromachined in silicon," Clinical Chemistry, vol. 40, no. 1, pp. 43-47, 1994.

[5] E. B. Arkilic, M. A. Schmidt, and K. S. Breuer, "Gaseous slip flow in long microchannels," IEEE Journal of Microelectromechanical Systems, vol. 6, no. 2, pp. 167-178, 1997.

[6] J. Pfahler, J. Harley, H. Bau, and J. Zemel, "Liquid transport in micron and submicron channels," Sensors and Actuators: A. Physical, vol. 22, no. 1-3, pp. 431-434, 1989.

[7] J. W. Hoyt and A. G. Fabula, "The effects of additives in fluid friction," US Naval Ordinance Test Station Report, 1964.

[8] G. P. Galdi and S. Rionero, "A note on the existence and uniqueness of solutions of the micropolar fluid equations," International Journal of Engineering Science, vol. 15, no. 2, pp. 105-108, 1977.

[9] H. Lange, "The existence of instationary flows in incompressible micropolar fluids," Archives of Mechanics, vol. 29, no. 5, pp. 741$744,1977$.

[10] G. Łukaszewicz, "Long time behavior of 2D micropolar fluid flows," Mathematical and Computer Modelling, vol. 34, no. 5-6, pp. 487-509, 2001.
[11] G. Łukaszewicz, "On nonstationary flows of incompressible asymmetric fluids," Mathematical Methods in the Applied Sciences, vol. 13, no. 3, pp. 219-232, 1990.

[12] V. A. Sava, "The initial-boundary-value problems in the theory of micropolar fluids," Zeitschrift für Angewandte Mathematik und Mechanik, vol. 58, no. 11, pp. 511-518, 1978.

[13] B. Yuan, "On regularity criteria for weak solutions to the micropolar fluid equations in Lorentz space," Proceedings of the American Mathematical Society, vol. 138, no. 6, pp. 2025-2036, 2010.

[14] E. Fernandez-Cara and S. Guerrero, "Local exact controllability of micropolar fluids," Journal of Mathematical Fluid Mechanics, vol. 9, no. 3, pp. 419-453, 2007.

[15] F. D. Araruna, F. W. Chaves-Silva, and M. A. Rojas-Medar, "Exact controllability of Galerkin's approximations of micropolar fluids," Proceedings of the American Mathematical Society, vol. 138, no. 4, pp. 1361-1370, 2010.

[16] R. Stavre, "The control of the pressure for a micropolar fluid, Dedicated to Eugen Soos," Zeitschrift für angewandte Mathematik und Physik, vol. 53, no. 6, pp. 912-922, 2002.

[17] S. K. Lakshmana Rao, "Stability of micropolar fluid motions," International Journal of Engineering Science, vol. 8, pp. 753-762, 1970.

[18] R. Russo and M. Padula, "A note on the bifurcation of micropolar fluid motion," Bollettino dell'Unione Matematica Italiana A, vol. 17, no. 1, pp. 85-90, 1980.

[19] T.-H. Hsu and S.-Y. Tsai, "Natural convection of micropolar fluids in a two-dimensional enclosure with a conductive partition," Numerical Heat Transfer, Part A: Applications, vol. 28, no. 1, pp. 69-83, 1995.

[20] S. K. Jena and S. P. Bhattacharyya, "The effect of microstructure on the thermal convection in a rectangular box of fluid heated from below," International Journal of Engineering Science, vol. 24, no. 1, pp. 69-78, 1986.

[21] V. U. K. Sastry and G. Maiti, "Numerical solution of combined convective heat transfer of micropolar fluid in an annulus of two vertical pipes," International Journal of Heat and Mass Transfer, vol. 19, no. 2, pp. 207-211, 1976.

[22] O. Aydin and I. Pop, "Natural convection in a differentially heated enclosure filled with a micropolar fluid," International Journal of Thermal Sciences, vol. 46, no. 10, pp. 963-969, 2007.

[23] S. Ye, K. Zhu, and W. Wang, "Laminar flow of micropolar fluid in rectangular microchannels," Acta Mechanica Sinica, vol. 22, no. 5, pp. 403-408, 2006.

[24] J. S. Hansen, P. J. Daivis, and B. D. Todd, "Molecular spin in nano-confined fluidic flows," Microfluidics and Nanofluidics, vol. 6, no. 6, pp. 785-795, 2009.

[25] J. Chen, J. D. Lee, and C. Liang, "Constitutive equations of Micropolar electromagnetic fluids," Journal of Non-Newtonian Fluid Mechanics, vol. 166, no. 14-15, pp. 867-874, 2011.

[26] J. L. Boldrini and M. A. Rojas-Medar, "On the convergence rate of spectral approximation for the equations for nonhomogeneous asymmetric fluids," Mathematical Modelling and Numerical Analysis, vol. 30, no. 2, pp. 123-155, 1996.

[27] A. C. Eringen, “Theory of micropolar fluids," Journal of Mathematical Mechanics, vol. 16, pp. 1-16, 1966.

[28] G. Lukaszewicz, Micropolar Fluids: Theory and Applications, Birkhäuser, Boston, Mass, USA, 1999.

[29] K.-H. Hoffmann, D. Marx, and N. D. Botkin, "Drag on spheres in micropolar fluids with non-zero boundary conditions for microrotations," Journal of Fluid Mechanics, vol. 590, pp. 319330, 2007. 
[30] E. DiBenedetto, Degenerate Parabolic Equations, Springer, New York, NY, USA, 1993.

[31] J. G. Heywood and R. Rannacher, "Finite-element approximation of the non-stationary Navier-Stokes problem. IV: error analysis for second-order time discretization," SIAM Journal on Numerical Analysis, vol. 27, no. 2, pp. 353-384, 1990. 


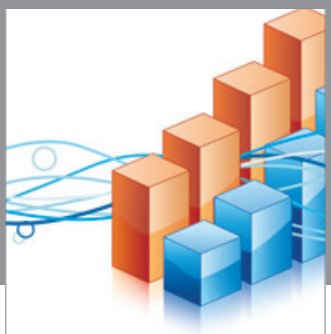

Advances in

Operations Research

vatem alat4

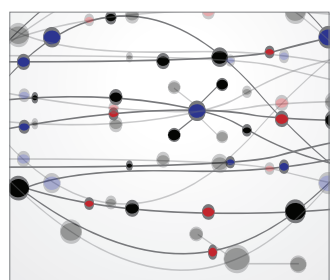

\section{The Scientific} World Journal
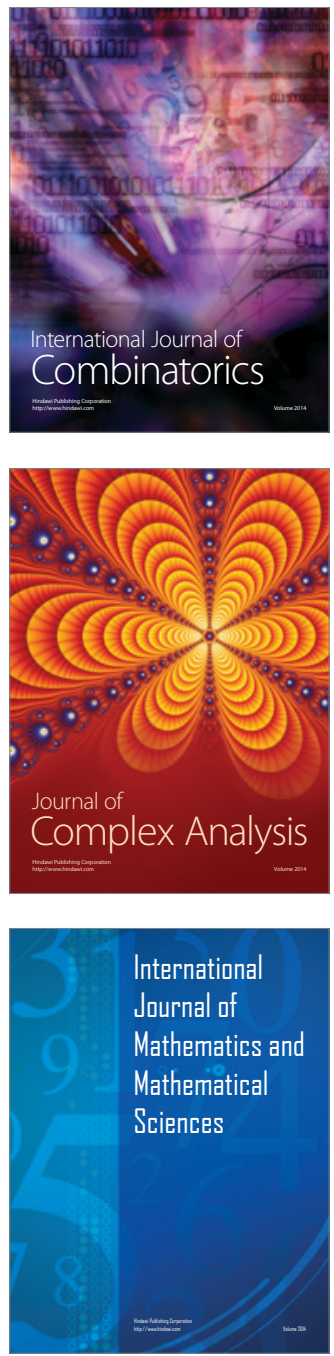
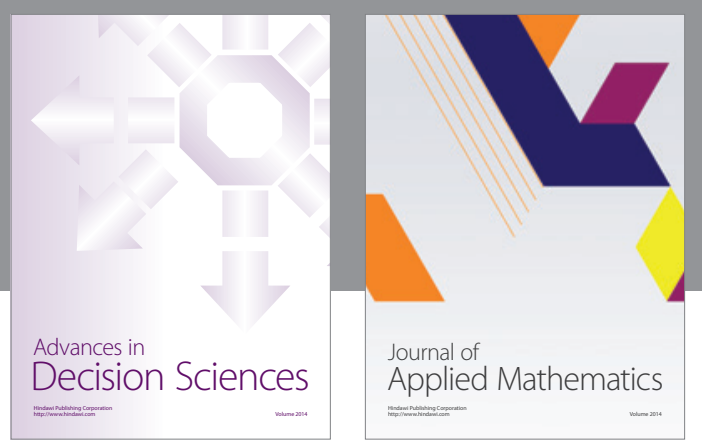

Algebra

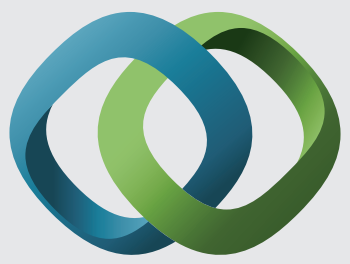

\section{Hindawi}

Submit your manuscripts at

http://www.hindawi.com
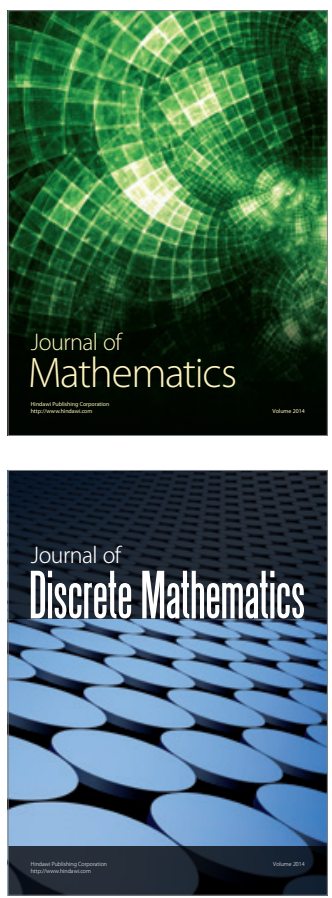

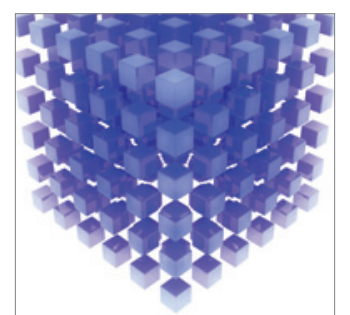

Mathematical Problems in Engineering
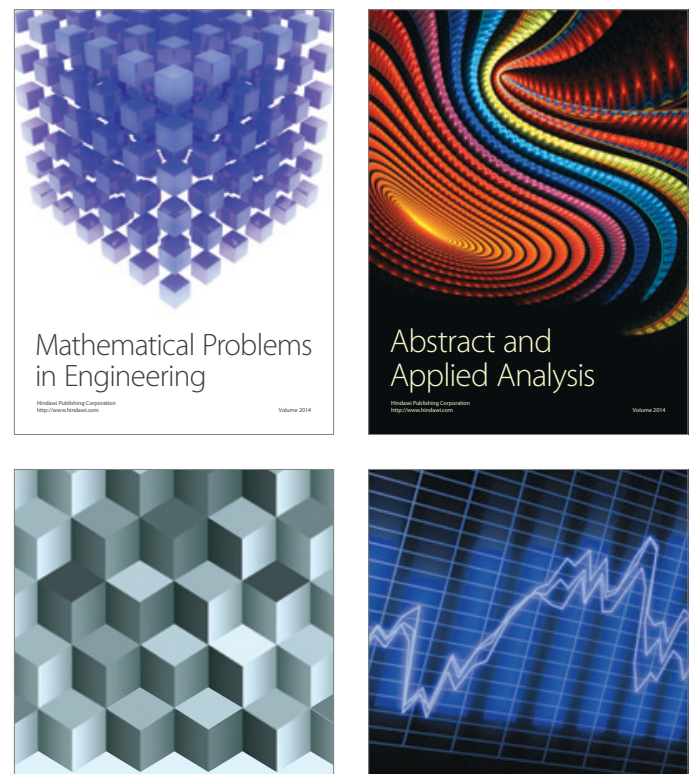

Journal of

Function Spaces

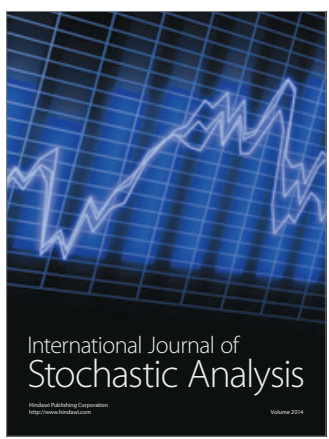

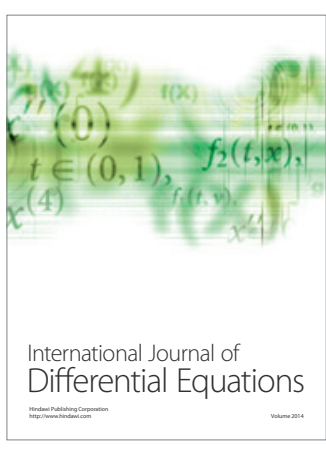
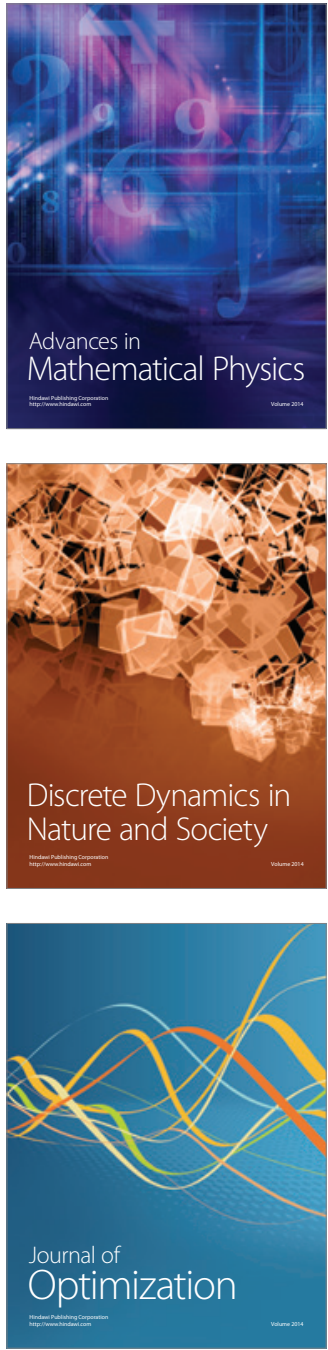